\title{
The interaction of round synthetic jets with a turbulent boundary layer separating from a rounded ramp
}

\author{
S. Lardeau $\dagger$ and M. A. Leschziner \\ Department of Aeronautics, Imperial College London, South Kensington Campus, London SW7 2AZ, UK
}

(Received 24 November 2010; revised 3 June 2011; accepted 6 June 2011; first published online 25 September 2011)

A computational large eddy simulation (LES) study is presented of the interaction between a turbulent boundary layer separating from a rounded ramp in a duct and a pair of spanwise-periodic, round synthetic jets, actuated upstream of the nominal separation line. Several scenarios are considered, for different injection angles and velocity ratios. In all cases, the actuation frequency corresponds to the sheddinginstability mode of the separated shear layer. Experimental data, available for both the baseline flow and one actuated configuration, are used to verify the validity of the computational solutions. The analysis includes a separation of coherent and stochastic contributions to the time-averaged statistics of the auto- and cross-correlations of the fluctuations. The control authority is examined by reference to the effects of the actuation on the size of the separated zone, the momentum thickness of the boundary layer, the velocity field, various turbulence quantities and phase-averaged properties. The study demonstrates that the principal aspect of the interaction, at mean-flow level, is an increase in mixing provoked by the formation of strong streamwise vortices away from the wall, the induction of much weaker streamwise vortices close to the wall, and the extra production of stochastic turbulence caused by unsteady straining. The coherent stresses arising from the periodic perturbations are high - typically 5 times the levels of the unperturbed flow - but only within about 5-7 diameters of the jet orifice, and 2 orifice diameters on each side of the jet, and these are dominant primarily in the outer parts of the boundary layer. Stochastic turbulence is also elevated, but more modestly. The global effect of the actuation is a reduction of $10-20 \%$ in the length of the separated region and $20-40 \%$ in the thickness of the reverse-flow layer, depending on the actuation scheme, counter-flow actuation being the most effective. This reduction is mainly associated with a delay in separation. These results highlight the need for synthetic jets to be placed close to the separation zone and for the inter-jet distance to be of order 5 or lower to achieve a high level of separation-control authority.

Key words: boundary layer separation, drag reduction, turbulence control

\section{Introduction}

A synthetic jet is an interacting train of vortex rings formed as a consequence of a fluid being ejected periodically through an orifice into a flow to be altered or

$\dagger$ Email address for correspondence: s.lardeau@imperial.ac.uk 
controlled in some sense. The expulsion phase is followed by a suction phase, the latter not necessarily anti-symmetric to the former, but constrained to result in a zero cycle-average mass flux through the orifice. During the expulsion stage, vorticity flux and associated circulation (the time-integral of the former) are ejected into the outer flow and propagate away from the orifice. In the subsequent suction phase, the fluid ingested into the orifice originates predominantly from the area close to the wall surrounding the orifice. Hence, this suction does not re-ingest more than a small fraction of the circulation ejected, and the result of this irreversible and asymmetric process is a net unsteady expulsion of vorticity. As the vortices propagate into the outer medium, they interact by merging and diffusing, so that the statistical state is a jet with broadly similar far-field characteristics to those of a continuous jet - albeit weaker in intensity (Smith \& Swift 2003).

In a practical setting, a synthetic jet is most frequently realised by oscillating the wall of a cavity bordering one side of the orifice, whilst on the other side, the vortical structures leaving the orifice interact with a cross-flowing boundary layer. Practical interest in synthetic jets is driven primarily by their potential to reduce or avoid flow separation from highly loaded aerodynamic surfaces, e.g. in high-lift flight conditions. While such a capability is also offered, in principle, by fence- or chevron-like vortex generators, synthetic jets allow on-demand separation control with the working fluid itself, but without the need for a mass source - a combination that is especially attractive in aeronautical applications.

The fluid-mechanics features of synthetic jets depend sensitively on several parameters, each set giving rise to a scenario that can differ substantially from others. The most important variable is the geometry of the orifice, ranging from a long slot to a circular hole. The behaviour of the ejected vortices and the manner in which they interact with the cross-flow are more complicated in the case of circular jets than slot jets, due to the more pronounced three-dimensionality of the processes in the former. The interaction is also weaker and more subtle. Other important variables include the position of the orifice(s) relative to the separation location, the angle of injection, the cavity and orifice-duct shape, the jet-to-outer-flow momentum ratio, the frequency of the injection, the temporal variation of the ejection/suction rate within the actuation period, the mean and turbulence properties of the boundary layer in the cross flow, the Reynolds number of the jet and the boundary layer and the details of the separated flow to be controlled. This complex multi-dimensional parameter space makes it extremely difficult to derive general conclusions and guidelines on the control authority of synthetic jets and the associated fluid mechanics. Rather, past studies provide no more than a sparse knowledge base that yields general pointers, but very little by way of quantitative data.

A fairly representative actuation scenario is shown in figures 1 and 2 - the outcome of one of the simulations discussed in $\S 4$, for a sinusoidal injection into a turbulent boundary layer with the maximum orifice-averaged ejection velocity equal to the free-stream velocity of the boundary layer, and an actuation frequency corresponding to a Strouhal number $S t_{d}=0.032$, based on the orifice diameter, $d$, and the maximum ejection velocity, $V_{r}$. The figures contain phase-averaged contours of the Laplacian of the pressure of the flow at 8 phase values, together covering the ejection phase of the full cycle, with injection starting at the phase value of zero.

The expulsion stroke first generates a vortex ring that is distorted by the cross-flow - in this case a boundary layer. The head of the ring is then lifted upwards by the Magnus force, while the ring is stretched by the shear strain in the boundary layer, the two effects causing the vortex ring to incline and elongate (Sau \& Mahesh 2008) 
(a)
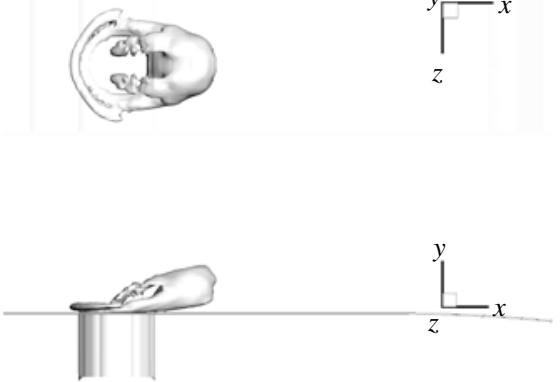

(c)
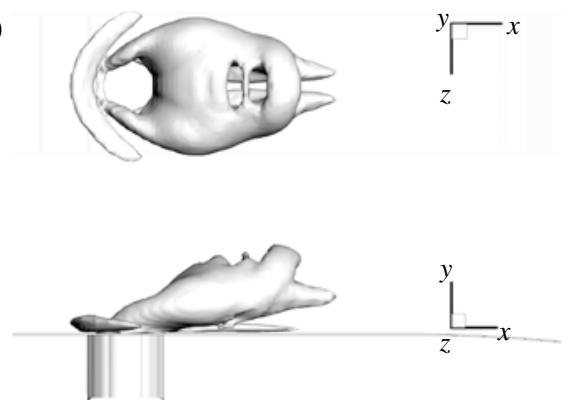

(b)
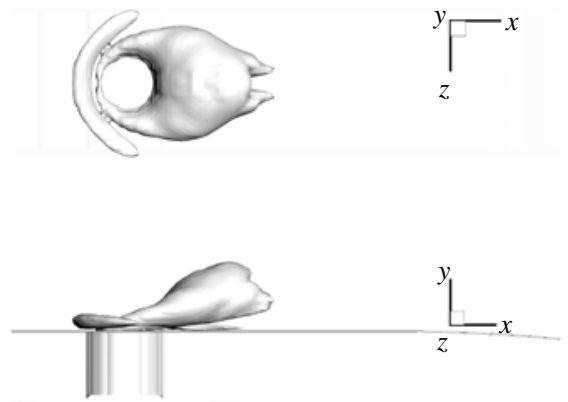

(d)
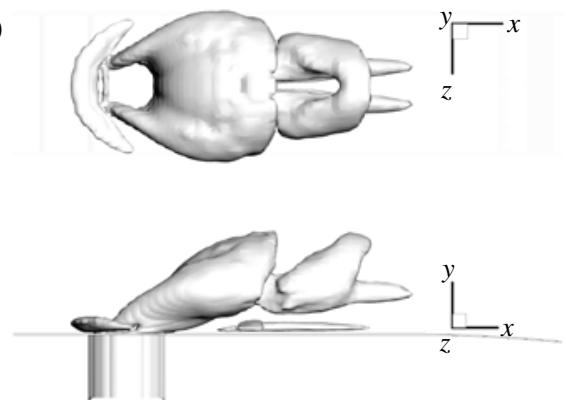

FIGURE 1. Iso-surfaces of the Laplacian of pressure representing the phase-averaged evolution of a round, turbulent synthetic jet at different phases within a single cycle. Case $C_{r e f}$ (see table 1). Expulsion phase: $(a) 0,(b) \pi / 8,(c) 2 \pi / 8$ and $(d) 3 \pi / 8$.

towards a hairpin-like shape. What is shown in figures $1(a)$ and $1(b)$ is a structure in the part of the ring in which the motion on the spanwise plane bisecting the orifice is clockwise, reinforced by the shearing motion of the boundary layer. Roughly halfway through the ejection phase, a pinching of the vortex ring occurs, terminating in the formation of a secondary inclined head vortex that is linked to the primary ring by arms with predominantly streamwise vorticity having the same sign as the vorticity in the legs of the hairpin-like structure. When the ejected vorticity flux is high, as is the case here, and is typical of low-frequency actuation, the vortex ring cannot contain all the vorticity that is ejected, due to saturation effects (Gharib, Rambod \& Shariff 1998), and the primary ring is followed by a tail of vorticity, as is well brought out in figure 2. Towards the end of the ejection phase, the jet structure is dominated by a pair of vortices, which are the legs of a hairpin-like structure connected far downstream by a head, no longer visible in figure 2 , which induces a downwash at its front. Thus, at this stage, the primary jet-induced motion is an upwash from the jet-centre region and a downwash in the outer regions, as is the case in a continuous jet in cross-flow. This causes an exchange - a mixing - of streamwise momentum by convective secondary cross-flow motion. Also seen in both figures 1 and 2 are thin secondary near-wall vortices, induced by the primary outer vortex as a consequence of the lateral inward motion close to the wall of sheared fluid towards the jet centre-plane. In the following suction stroke (not included), the tails of the hairpin-like structure are pulled back into the orifice, elongating this structure further. Otherwise, the suction stroke has an effect 
(a)
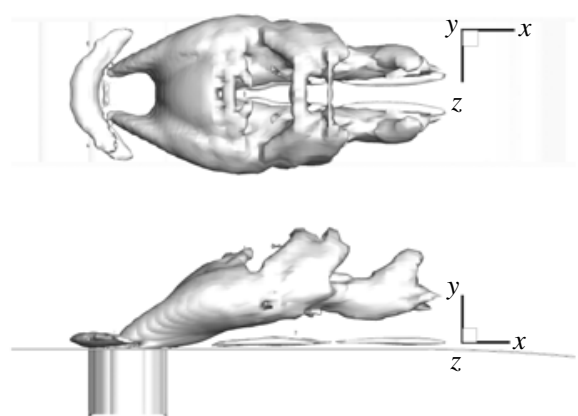

(c)
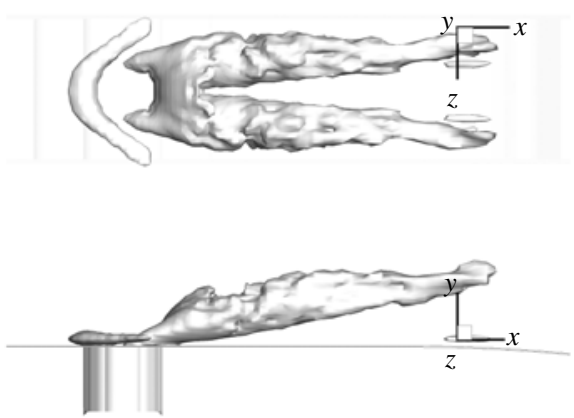

(b)
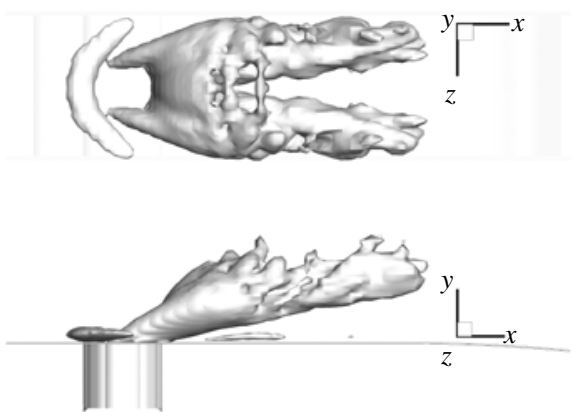

$(d)$
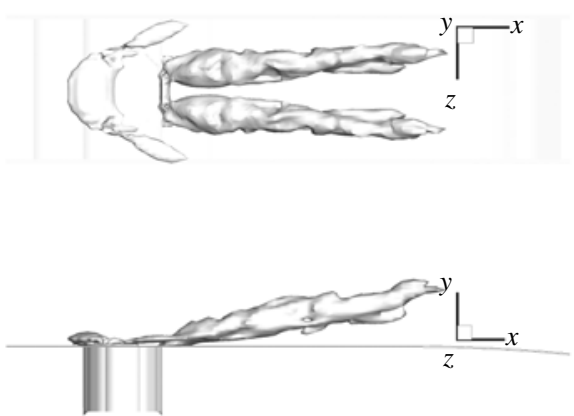

FIGURE 2. The same as figure 1 , for $(a) 4 \pi / 8,(b) 5 \pi / 8,(c) 6 \pi / 8$ and $(d) 7 \pi / 8$.

very similar to a sink flow, wherein the fluid that is drawn into the orifice emanates predominantly from the near-wall layer (Gordon \& Soria 2002).

A time-averaged view of the present flow is given in figure 3 by way of contours of streamwise vorticity, and isolines of streamwise and spanwise velocity. This representation brings out statistical similarities between a synthetic jet and a steady jet in a cross-flow. In particular, the downstream state is characterised by a pair of streamwise vortices, corresponding to some of the phase-averaged features observed earlier. One important distinction from a continuous jet is that the time-averaged flow upstream of the jet is marked by a streamwise acceleration, rather than deceleration, because the net zero-mass-flow constraint through the orifice forces the flow into the cavity on the upstream edge of the jet. The jet is seen to induce significant lateral motions, of order $10 \%$ of the free-stream velocity, and strong distortions in the streamwise velocity. The latter indicate a deceleration below and within the region of strong streamwise vorticity, an acceleration of fluid within a tongue above this region - indicative of the trajectory of the jet - and by acceleration in the near-wall region downstream of the jet, as the fast outer fluid is being transported towards the wall.

Past research on synthetic jets, and the related literature, are extensive, but dominated by studies on slot jets, especially in respect of separation control. The state of the art in this area is best exemplified by the experimental work of Greenblatt et al. (2006) for slot-jet injection into a boundary layer separating from a dune-shaped hump, and a number of computational studies corresponding to this experimental geometry, documented in Rumsey et al. (2006), You, Ham \& Moin (2008), Krishnan, Squires \& Forsythe (2006) and Avdis, Lardeau \& Leschziner (2009). Related simulations 

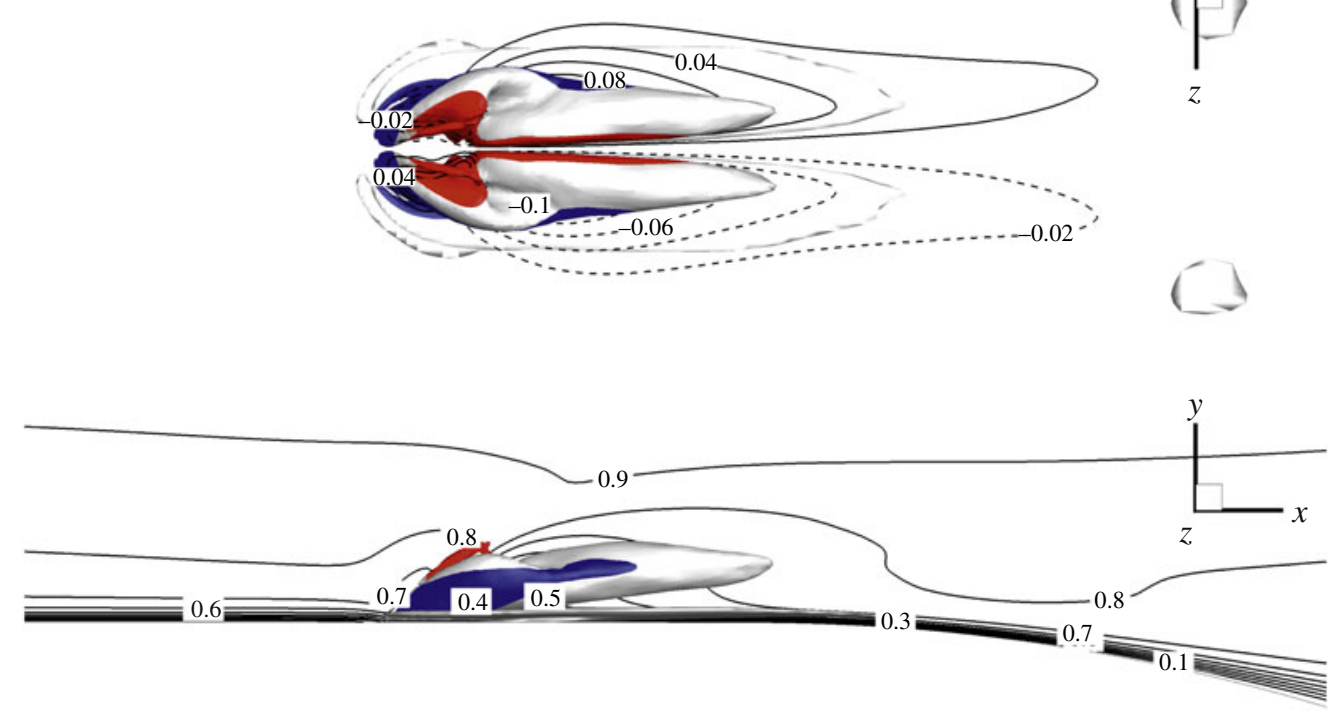

FIgURE 3. Time-averaged representation of the jet shown in figures 1 and 2. Isosurface identifying the streamwise vorticity $\omega_{x}= \pm 1 U_{i n} / H$; coloured portions of isosurface identify, in red, positive wall-normal velocity and, in blue, negative velocity; contour lines in the top view represent the spanwise velocity $W / U_{\text {in }}$ at $y / H=0.05$ from the wall; lines in side view represent $U / U_{\text {in }}$ on the jet centre-line.

for slot-jet injection into boundary layers separating from curved backward-facing ramps are those of Neumann \& Wengle (2004) and Dandois, Garnier \& Sagaut (2007). Most of the studies on slot jets focus on global characteristics, e.g. the reduction in recirculation length depending on injection intensity and frequency, and the relationship of this global behaviour to the phase-averaged characteristics and their interaction with the turbulence field. In a few cases, e.g. Dejoan \& Leschziner (2004) and Pamart et al. (2010), attention is directed towards the coupling between the actuation frequency and natural instability modes in the separated shear layer.

There are a number of reasons for the above-described bias towards slot jets, which are discussed by Leschziner \& Lardeau (2011). Among them is the fact that slot jets are held to afford substantially greater control authority on the separation process, due to their much larger cross-flow extent and the mass and vorticity ejected. Another reason is that the statistical properties of slot jets are adequately described, in experiments, by the conditions on the spanwise mid-slot plane, and indeed fully described on any spanwise plane in computational studies in which statistically spanwise homogeneity is imposed, as is the case in most such studies, whether using large eddy simulation (LES) or Reynolds-averaged Navier-Stokes (RANS) methods. From a computational point of view, slot jets also pose fewer resolution problems that arise from the major scale disparity between the orifice and the flow to be controlled, due to the predominantly two-dimensional conditions of the near-field flow close to the orifice.

Within the area of round synthetic jets, there have been a number of experimental as well as computational studies examining the behaviour of such jets in stagnant 
surroundings. A brief discussion by Saffman (1981), under the heading Dynamics of Vorticity, of a periodically ejected train of interacting vortices, visualised experimentally by Glezer (1981), is perhaps the earliest account pertinent to the fluid mechanics of a synthetic jet discharged into stagnant surroundings. A detailed discussion of the fluid mechanics of synthetic jets in stagnant media is given by Glezer \& Amitay (2002), based on work by Smith \& Glezer (1998). More recent experimental studies, exploring mainly basic aspects of the formation of the jets and/or statistical properties of synthetic jets relative to continuous jets, are those of Zhong et al. (2007), Smith \& Swift (2003) and Cater \& Soria (2002), with a corresponding LES realisation of the last reported by Wu \& Leschziner (2009). Simulation studies by Rizzetta, Visbal \& Stanek (1999), Raju et al. (2009), Aram, Mittal \& Cattafesta (2010) and Mittal \& Rampunggoon (2002), for two-dimensional or high-aspect-ratio slots jets at low Reynolds numbers, are also noteworthy, in so far as they identify the importance of including the cavity in the computational realisation - in particular, the effect of the cavity flow on the near-field vortical structure during the ejection phase and the non-uniform conditions across the orifice. While these investigations bring to light some fundamentally interesting consequences of the manner in which the ejected vortex rings form, propagate and interact to form a jet, they are only weakly pertinent to the subject of this paper, simply because the fluid mechanics of such jets differ greatly from those in a cross-flow. In particular, the manner in which a jet and the vortical structures therein propagate in stagnant surroundings, beyond the immediate vicinity of the orifice, are dictated purely by mutual interactions among the vortical structures expelled and the motions they induce. Moreover, the phase- and time-averaged states of such jets are two-dimensional, and the control of the flow into which these jets are injected is not a relevant issue.

As noted already, few studies target the interaction of round synthetic jets with a cross-flowing boundary layer, and all but one are for attached conditions - the one exceptional study involving a boundary layer separating in a laminar state from a flat plate due to contouring of an opposing wall (Ozawa, Lesbros \& Hong 2010).

Three studies that examine the formation and development of round laminar jets injected into laminar boundary layers are those of Jabbal \& Zhong (2008), Zhou \& Zhong (2009) and Sau \& Mahesh (2008), the former experimental and the other two computational. These investigations, especially the latter, are remarkable in so far as they bring out with striking clarity the frequency-dependence of the shape and evolution of the vortex rings expelled by the actuation, and of the associated secondary vortical structures and velocity fields that are induced. They also highlight some of the basic fluid-mechanic characteristics, corresponding to those discussed earlier by reference to figures 1 and 2, although the level of correspondence is necessarily diminished by the absence or presence of turbulence. In particular, Zhou \& Zhong (2009) observe two types of vortical structures, depending on the injection conditions: hairpin structures, at relatively low frequency, and tilted vortex rings at higher frequency. A variety of induced vortices are identified, and these are shown to depend on the injection frequency.

The interaction between round (or square) jets and attached turbulent boundary layers has been studied experimentally by Gordon \& Soria (2002), Schaeffler \& Jenkins (2006), Zaman \& Milanovic (2002) and Garcillan et al. (2006), while computational investigations include those of Ravi, Mittal \& Najjar (2004), Dandois, Garnier \& Sagaut (2006) and Wu \& Leschziner (2009), the first restricted to verylow-Reynolds-number laminar conditions and the last two being fully turbulent and corresponding to the experiments of Schaeffler \& Jenkins (2006) and Garcillan et al. 
(2006), respectively. Most of these studies are moderately pertinent to the present paper, in so far as they quantify the effect of the actuation on the boundary layer downstream of the orifice, within a region that lies upstream of the separation line, as is the case in the flow which is the subject of this study. The studies reveal, collectively, a wide range of interesting features, the most important common observations being: the narrowness of the lateral domain within which the jet modifies the base flow, extending to only around one orifice diameter or two on either side of the jet centre-plane, the presence of two major time-averaged vortex pairs, one in the outer portion of the boundary layer, reflecting the trajectory of the jet, and another counter-rotating pair close to the wall; the occurrence of a mild acceleration of the streamwise flow near the wall, due to streamwise momentum being transported towards the centre-plane by the near-wall vortex pairs; the creation of substantial distortions in the phase-averaged velocity profiles in the near-orifice field, associated with near-wall flow acceleration and the presence of a distinct velocity maximum in the region in which the head of ejected vortex induced flow acceleration; a fairly rapid decay of the control effect beyond around $x / d=5$, following a modest increase of about $30 \%$ in the boundary-layer displacement and momentum thicknesses, and the presence of strong distortions in the velocity field across the orifice, suggesting that it is imperative to resolve the flow below the orifice.

The above review serves to demonstrate that there has been virtually no serious attempt so far to examine the interaction between round synthetic jets and separating turbulent boundary layers. Not only is this configuration of considerable fundamental interest, it is also practically important, because matrices of circular orifices, as opposed to long slots, permit higher structural integrity to be maintained in patches of highly loaded aerodynamic surfaces. The present paper thus presents a study of the interaction between a pair of round synthetic jets with a turbulent boundary layer separating from a rounded backward-facing ramp. The study applies LES to a number of combinations of injection parameters for one and the same ramp geometry, among them injection intensity, pitch angle and frequency. For two sets of conditions, including the unforced baseline flow, the computations are compared with extensive laser Doppler anemometry (LDA) and particle image velocimetry (PIV) data of Zhang \& Zhong (2010). Particular attention is focused on basic fluid-mechanic characteristics, including a distinction between coherent and stochastic contributions to the turbulence fields.

\section{The flow configuration}

The computational geometry under consideration is shown in figure 4. This corresponds closely to the experimental configuration of Zhang \& Zhong (2010). The only major difference is that the experimental high-aspect-ratio duct was here approximated by a spanwise-periodic slab with planes separated by 3.2 ramp heights, $H$. The duct height upstream of the ramp is $8.52 \mathrm{H}$, and this was included in the simulation in its entirety. The computational domain includes two circular orifice ducts of diameter $d=0.16 \mathrm{H}$, separated by $10 d$, and the associated (separate) cylindrical actuation cavities of diameter $D=3.7 \mathrm{H}$. The two jets are discharged $4 d(0.64 \mathrm{H})$ upstream of the curved ramp, an arrangement dictated by experimental constraints. In all above respects, the computed and experimental configurations are identical.

A turbulent boundary layer separates from the backward-facing rounded ramp at approximately $0.9 \mathrm{H}$ downstream of the start of the ramp. The ramp geometry is based on that used originally by Song \& Eaton (2004), but was adapted (by increasing 


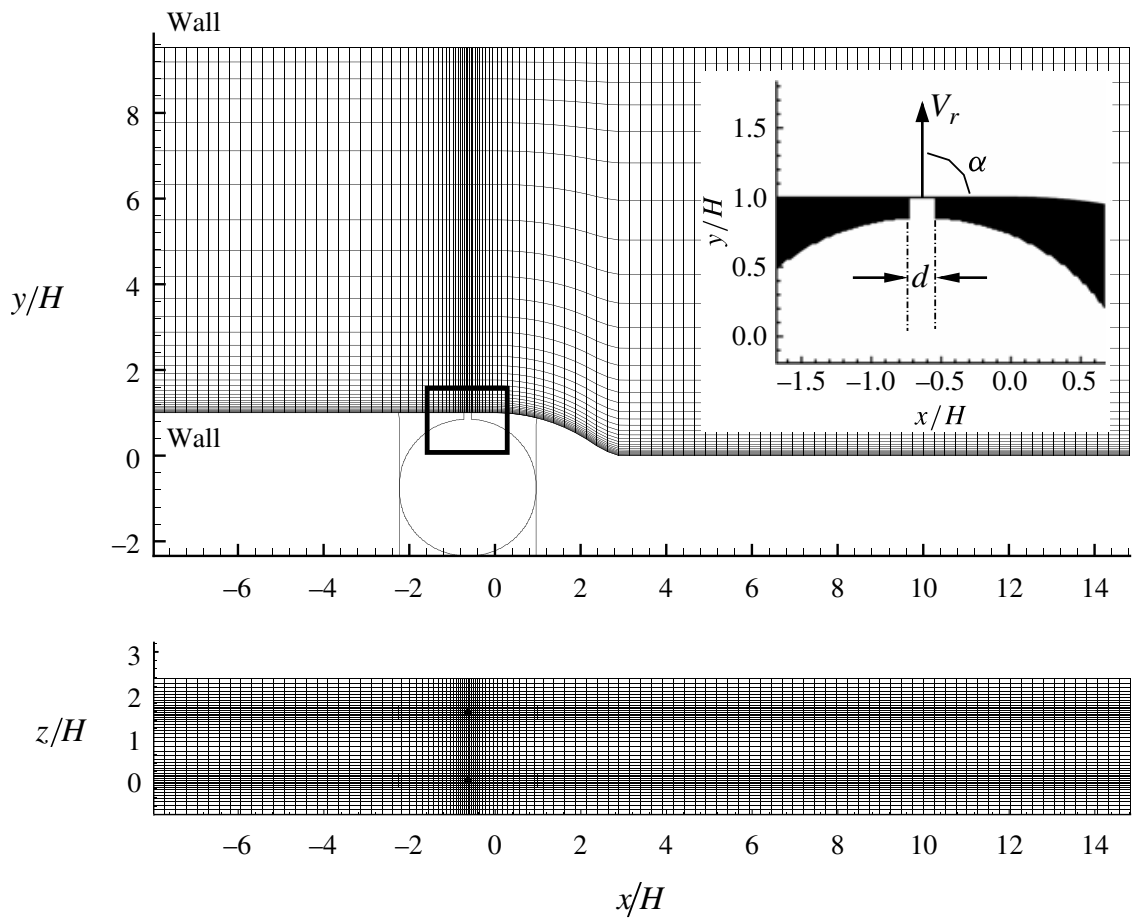

FIGURE 4. Solution domain and reduced numerical mesh: only every fourth grid line is shown, and the grid embedded in the two circular cavities and orifices is omitted.

the step height) in order to increase the size of the separation bubble, which was regarded to be too weak in Song \& Eaton's experiment. This adaptation was undertaken interactively with the experimental effort, based on preliminary RANS and LES computations.

The ramp shape is described by the following three relations, with the origin $x / H=0$ being the upstream edge of the ramp:

$$
\begin{gathered}
y_{\text {wall }}=\left(1-R_{1}\right)+\sqrt{R_{1}^{2}-x^{2}} \quad \text { for } 0<x / H<2.3, \\
y_{\text {wall }}=y_{2}-\sqrt{\frac{R_{1}^{2}}{4}-\left(x_{2}-x\right)^{2}} \text { for } 2.3<x / H<2.835, \\
y_{\text {wall }}=R_{2}-\sqrt{R_{2}^{2}-(3-x)^{2}} \quad \text { for } 2.835<x / H<2.937,
\end{gathered}
$$

with $R_{1}=4.03, R_{2}=0.333, x_{2}=3.449$ and $y_{2}=1.936$. The distance from the bottom wall $y_{w}=\left(y-y_{\text {wall }}\right)$ is also used throughout the text.

The Reynolds number, based on the ramp height $H$ and the inlet free-stream velocity $U_{\text {in }}$, is 13700 . In the experiment, the boundary layers on both walls evolve over a long stretch of duct, with the ramp being around $25 \mathrm{H}$ long, and attain a state very close to that of a canonical, turbulent flat-plate boundary layer, as verified by LDA measurements. At $x / H=-8$, the computational inlet, the momentum-thickness Reynolds number is $R e_{\theta}=1160$, and the boundary-layer thickness is $\delta_{99}=0.9 H$. In the computations, the turbulent boundary layers on the upper and lower walls of the duct at the domain inlet were imposed using data from a precursor simulation, 

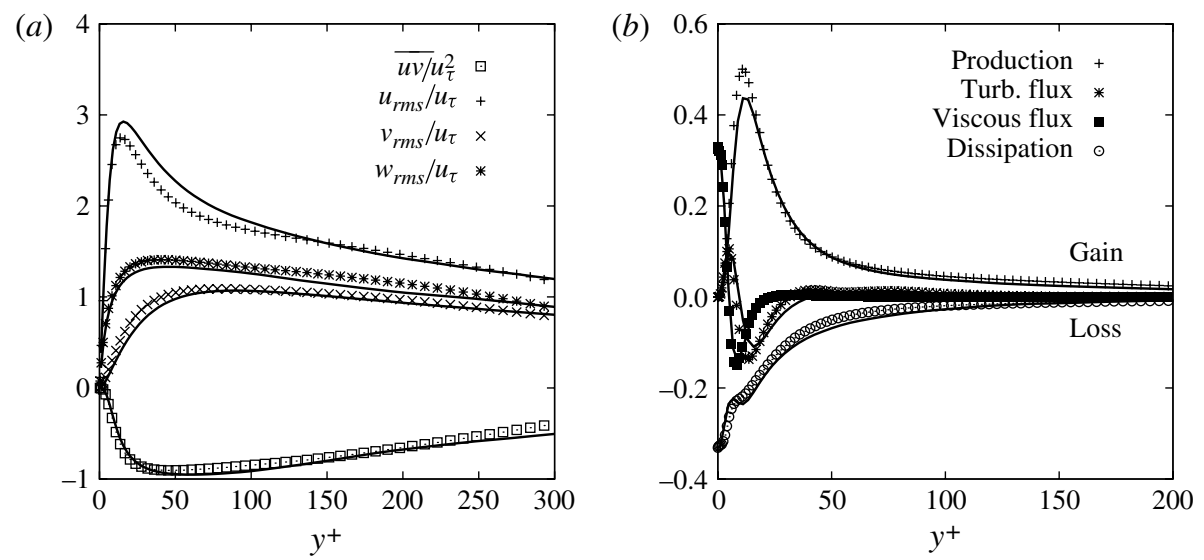

FIGURE 5. State of the turbulent boundary layer at $x / H=-4:(a)$ turbulence-intensity and shear-stress profiles; $(b)$ budgets for the streamwise Reynolds stress $\overline{u u}$. Symbols, DNS results from Jiménez et al. (2010); solid lines, present LES results.

performed with the aid of a recycling method (Lund, Wu \& Squires 1998), for a stretch of a boundary layer developing in the actual channel. At a streamwise position at which $R e_{\theta}=1160$, every realisation of the velocity field was stored over a period of $1000 H / U_{i n}$. This data set was then used to explicitly prescribe the inlet conditions. Comparisons between boundary-layer data returned by the present LES at $x / H=-4$ and direct numerical simulation (DNS) results of Jiménez et al. (2010), for a similar Reynolds number, $R e_{\theta}=1100$, are shown in figure 5 . Agreement is seen to be close, and this demonstrates the high-fidelity representation of the boundary layer approaching the ramp.

The synthetic jets are actuated sinusoidally and in phase. This is realised, in the experiment (Zhou \& Zhong 2009), by a shaker-piston mechanism to which one sidewall of each cavity is attached. As the area of the cavity wall is very large, relative to the orifice area, the displacements involved are minimal relative to the cavity depth. Thus, in the computation, the motion of the piston is represented by a weak sinusoidal transpiration on the appropriate sidewall in the $z$-direction:

$$
V_{\text {piston }}=V_{0} \sin \left(2 \pi \frac{t}{T}\right)
$$

where $V_{0}$ is the maximum sidewall velocity and $T$ the period of the actuation. The jet velocity can be deduced from (2.2) using the simple incompressible-flow relationship:

$$
V_{j e t}=\frac{V_{\text {piston }}}{d^{2} / D^{2}} .
$$

The mass-averaged exit velocity over the ejection phase may be computed from

$$
V_{\text {exit }}=\frac{2}{T} \int_{0}^{T / 2} V_{r} \sin \left(2 \pi \frac{t}{T}\right) \mathrm{d} t,
$$

where $V_{r}$ is the maximum value of $V_{j e t}, V_{r}=\pi / 2 V_{\text {exit }}$. The Reynolds number, based on $V_{r}$ and $d$, is $R e_{j e t}=2900$, which is high compared to other studies (e.g. Sau \& Mahesh 2008). 


$\begin{array}{lcccccccc}\text { Case } & S t_{H} & V_{r} / U_{i n} & \alpha & T & L / d & V_{\text {exit }} / U_{\text {in }} & c_{\mu}(\%) & \text { Symbol } \\ \text { Baseline LES } & 0 & 0 & 0 & 0 & 0 & 0 & 0 & - \\ \text { Refined LES } & 0 & 0 & 0 & 0 & 0 & 0 & 0 & -- \\ \text { Fine LES } & 0 & 0 & 0 & 0 & 0 & 0 & 0 & -- \\ C_{r e f} & 0.2 & 1 & 90^{\circ} & 5.16 & 5.2 & 0.32 & 0.16 & -- \\ C_{V_{r}-0.5} & 0.2 & 0.5 & 90^{\circ} & 5.16 & 2.6 & 0.16 & 0.08 & + \\ C_{V_{r}+0.5} & 0.2 & 1.5 & 90^{\circ} & 5.16 & 7.74 & 0.48 & 0.24 & \times \\ C_{\alpha-45} & 0.2 & 1.0 & 45^{\circ} & 5.16 & 5.2 & 0.32 & 0.16 & \mathbf{\square} \\ C_{\alpha+45} & 0.2 & 1.0 & 135^{\circ} & 5.16 & 5.2 & 0.32 & 0.16 & \circ\end{array}$

TABLE 1. Flow conditions examined.

The normalised stroke length, $L / d$, corresponding to the length column of fluid ejected during the outstroke, is:

$$
\frac{L}{d}=\frac{V_{e x i t} T / 2}{d} .
$$

There are several ways in which the jet velocity is used to express the relative intensity of the actuation. The most popular parameter in the relevant literature is the momentum coefficient, $c_{\mu}$ :

$$
c_{\mu}=\frac{\rho_{j} V_{e x i t}^{2} d^{2}}{\rho_{\infty} U_{i n}^{2} L_{r e f} L_{z}},
$$

where $L_{\text {ref }}$ is taken here as the length of the recirculation region for the baseline case $\left(L_{r e f} / H=3.3\right)$, and $L_{z}$ is the spanwise width of the domain. In the present case, density is assumed to be constant throughout.

In what follows, five actuation scenarios are examined, and these are summarised in table 1. The first three entries are distinguished by the use of three different grids for the unforced baseline case; the difference will be explained in the next section. Case $C_{r e f}$ is the principal (reference) actuated case, for which experimental data are available. Two parameters are varied relative to that case: the pitch angle $\alpha$ (three angles from $45^{\circ}$ to $135^{\circ}$, with respect to the streamwise direction) and three velocity ratios $V_{r}$ (from 0.5 to 1.5 times the free-stream velocity $U_{i n}$ ). Several other control parameters, including the injection frequency and the shape of the duty cycle, have also been investigated, but in none of these variations was the effect on the separation drastically different from the reference case, and the results derived are not, therefore, included in this paper. Attention is drawn to the notation in table 1 that is used to identify different actuation scenarios. Specifically, the subscripts of the last four cases indicate variations in either injection velocity or angle relative to the reference case. Thus, $C_{\alpha+45}$ indicates that the injection angle was increased, relative to the reference of $90^{\circ}$, by $45^{\circ}$.

It is not claimed that the present study explores, in any systematic sense, the wide parameter space that might be of interest in a practical context. Rather, the main objective is to gain insight into the fluid-mechanic modifications associated with varying these parameters within reasonable bounds. 


\section{The computational approach}

The implicitly filtered LES momentum and continuity equations for incompressible flow were solved over a general non-orthogonal, boundary-fitted, multi-block finitevolume mesh, supplemented by the immersed boundary method (Udaykumar et al. 2001) to cater specifically to the complexities posed by the round orifices and the cavities. In total, the default mesh covering the duct-flow domain contained around 11 million nodes, while each cavity was meshed with a further 0.8 million nodes. The sensitivity to the resolution is addressed below. The variables are stored in a co-located manner. The solution is based on a fractional-step time-marching method, with the time derivative approximated by a third-order Gear scheme (Fishpool \& Leschziner 2009). The fluxes are approximated by second-order centred approximations. Within the fractional-step algorithm, a provisional velocity field results from advancing the solution with the flux operators. This is then corrected through the pressure field by projecting the provisional solution onto a divergence-free velocity field. To this end, the pressure is computed as a solution to the pressure-Poisson problem with the aid of a V-cycle multigrid scheme. In order to suppress unphysical oscillations, associated with pressure-velocity decoupling, a practice equivalent to that introduced in Rhie \& Chow (1983) is adopted. Fishpool \& Leschziner (2009) demonstrate that the loss of accuracy associated with the smoothing introduced by this practice is minimal.

While a grid of 11 million nodes, covering the main flow domain, might be regarded as modest, this needs to be seen against the relatively low Reynolds number of the flow simulated, and also the very long simulations that needed to be performed to achieve convergence, not only of the time-averaged quantities, but also the phaseaveraged quantities - and this subject to spanwise inhomogeneity preventing spanwise averaging.

To explore the sensitivity to grid resolution, two additional simulations for the baseline case, referred to as 'Refined LES' and 'Fine LES' in table 1, respectively, were performed. With the 'Refined LES' grid, the orifice-oriented refinement (figure 4) was removed, the spanwise grid was made uniform and the separated region (from $x / H=0$ to $x / H=4$ ) was resolved using 130 points in the streamwise direction, compared to 75 points for the original mesh. With the 'Fine LES' grid, the overall number of nodes was more than doubled to 24 million nodes, with 280 nodes covering the streamwise extent of the separated region.

In all three grids, the first grid-cell centre is located below $y^{+}=1$ at the bottom wall, whether $y$ is scaled with the local or inflow friction velocity. The maximum streamwise cell distance, $\Delta x^{+}$, occurs close to the computational inlet and is around 35 in the fine grid and 50 in the refined grid. This value drops to below 20 and 10 , respectively, downstream of $x / H=0$, with values of order 5 within the separation zone. The maximum spanwise cell dimension, $\Delta z^{+}$, is 10 for both the fine and refined simulations. For the 'Baseline LES' case, with non-regular spanwise distribution, $\Delta z^{+}$ varies from 17 between the two orifices to 5 close to the orifice centres, with $16 \times 16$ cells covering each orifice. Downstream of separation, the ratio of maximum cell dimension to the Kolmogorov length (derived from the dissipation rate as part of the budget) is of order 7 for the fine grid and 11 for the refined grid.

The boundary layer on the upper wall was covered by a coarser grid than that on the lower wall, with the conditions between the wall and the wall-nearest $y^{+}$ location of around 25 being covered by a log-law wall function, and with about 17 grid nodes covering the boundary-layer thickness. This choice is justified by the fact that the upper boundary layer is far away from the region of interest, is only subjected to a weak adverse pressure gradient and is always fully attached. An entirely 
analogous approach was taken by Fröhlich et al. (2005) when simulating the flow at a similar Reynolds number in a much tighter channel with massive separation behind streamwise periodic hill-shaped obstructions on the lower wall. In that case, this choice was made after extensive grid-sensitivity trials, and it is now well established that the resulting solution, including budgets, is fully trustworthy.

The subgrid-scale (SGS) stresses were approximated using either the dynamic Smagorinsky model of Germano et al. (1991) and Lilly (1992) or the mixed-time scale model of Inagaki, Kondoh \& Nagano (2005), the latter being local, involving no averaging and hence being computationally cheaper. The dynamic model was used for all actuated cases, and for the default baseline case, while the mixed-time scale model was adopted in the additional two grid-sensitivity simulations for the baseline case. The rationale of using the simpler mixed-time scale model for the grid-sensitivity study is rooted primarily in the observation that this model gave somewhat lower time-averaged SGS viscosity values than the dynamic model, and thus allowed any grid-sensitivity specifically associated with variations in resolution to emerge with greater clarity. Moreover, this model is computationally cheaper, which was especially advantageous in the fine-grid simulation. The decision to use the dynamic Smagorinsky model for the actuated cases - and also, consistently, for the baseline case computed with the same grid - reflected the prevailing view that this model is well founded, on physical grounds, especially for highly perturbed turbulence remote from equilibrium, and thus preferable to non-dynamic implementations. For all three meshes examined, the time-averaged subgrid-scale viscosity was found to be below $30 \%$ of the fluid-viscosity values in the separated region, declining to below $20 \%$ with the finest mesh. This compares with the ratio of the RANS-equivalent turbulent viscosity to fluid viscosity, the former estimated from the RANS $(k-\epsilon$ model) approximation $0.09 \rho k^{2} / \epsilon$, of $200-300$ in the separated zone. In the boundary layer approaching separation, the maximum subgrid-scale viscosity was well below $10 \%$ of the fluid-viscosity value. Consistent with the low values of the modelled viscosity, with both SGS models, the sensitivity of the quantities of interest to the grid have been observed to be marginal, including in respect of the terms contributing to the turbulence-energy budgets. This will be illustrated in figures to follow. In particular, figure 7 contains results obtained with all three grids for the skin friction and wall-pressure coefficients, the discussion of which is deferred to $\S 4$.

The simulations were performed with an average time step of $\Delta t=2 \times 10^{-3} H / U_{\text {in }}$ for the baseline case, and $\Delta t=6 \times 10^{-4} H / U_{\text {in }}$ for the actuated cases, corresponding to a maximum cell-Courant-Friedrichs-Lewy value of around 0.5 . In the latter cases, simulations were run for 50 time units (or about 10 injection cycles) before starting any statistical sampling. The flow was then computed for a further 230 time units (more than 40 injection cycles), during which data for various statistical properties were gathered. Statistical convergence was increased by using all symmetry planes possible (three spanwise planes for the actuated cases, and full spanwise averaging for the baseline flow). For each actuated case, phase-averaged data (denoted by $\langle\cdot\rangle$ ) were obtained, in addition to time-averaged fields (denoted by -). To this end, each period was divided into 16 phase bins, within each of which averaging was performed over all time steps in that bin. This bin-internal averaging further enhanced the convergence of the phase-averaged data. A quantitative indication of convergence is conveyed by the observation that the streamwise stress formed with the periodic component of the motion and obtained after 30 and 40 periods did not change by more than $5 \%$. As will be shown later, phase-averaged data form smooth fields and profiles, providing further indications that the data are well converged. 
To distinguish periodic (coherent) from stochastic contributions to time-averaged statistics, a triple decomposition (e.g. Hussain 1983) is adopted:

$$
f(\boldsymbol{x}, t)=\bar{f}(\boldsymbol{x})+\tilde{f}(\boldsymbol{x}, t)+f^{\prime}(\boldsymbol{x}, t),
$$

where $\bar{f}$ is the time-average value, $\tilde{f}$ is the periodic (or non-stochastic) perturbation and $f^{\prime}$ denotes the stochastic component.

The phase-average value is thus:

$$
\langle f\rangle=\bar{f}(\boldsymbol{x})+\tilde{f}(\boldsymbol{x}, t) .
$$

This is obtained directly from the simulation, allowing the stochastic component to be evaluated. Also, given the time-mean value, the coherent component can readily be obtained.

The momentum equations corresponding to the mean-flow component of the decomposition in (3.1) are:

$$
\frac{\mathrm{D} U_{i}}{\mathrm{D} t}=-\frac{\partial P}{\partial x_{i}}+\frac{1}{R e} \frac{\partial^{2} U_{i}}{\partial x_{j} \partial x_{j}}+\frac{\partial}{\partial x_{j}}\left(-\overline{\tilde{u}_{i} \tilde{u}_{j}}-\overline{u_{i}^{\prime} u_{j}^{\prime}}\right) .
$$

Equation (3.3) differs from the momentum equation obtained with the classical Reynolds decomposition only by the extra term $\left(-\left(\partial / \partial x_{j}\right)\left(\tilde{\tilde{u}}_{i} \tilde{u}_{j}\right)\right)$, which is related to the interactions of the periodic fluctuations among themselves. Separation can be delayed either by an increase of the stochastic or the periodic component. These two mechanisms will feature in the discussion to follow.

\section{Results}

\subsection{The baseline flow}

This section provides a discussion of the unforced baseline flow, so as to set the appropriate background against which to view the effects of the synthetic jets. Although it is not the primary target of the present study, the flow is interesting and challenging in its own right, and it deserves to be analysed in some detail, as is demonstrated in Song \& Eaton (2004). The challenge arises primarily from the fact that separation takes place from a gently curving surface and is thus characterised by a convoluted, time-varying and patchy separation behaviour, and by statistical properties around the time-mean separation line that differ drastically from those of a boundary layer separating cleanly from a sharp edge.

First, an instantaneous visualisation of the flow is shown in figure 6. Isocontours of $u=0$ are used to identify reverse-flow zones. Upstream of the separation, which occurs around $x / H=0.9$, the boundary layer is fully turbulent (cf. figure 5). The separation line is not well defined, and the 'jitter' of the isosurface corresponds roughly to the spanwise spacing, $\Delta z^{+}=80$ (figure $7 d$ ), of the streamwise streaks present in the incoming, attached boundary layer. Also visible are large 'blobs' of attached flow in the middle of the separation region. Unlike separation from a sharp step, where large spanwise-homogeneous structures are observed, the behaviour is here more akin to that of incipient, intermittent separation, and is not accompanied by distinct Kelvin-Helmholtz-type vortices. Reattachment is very indistinct and intermittent, occurring around $x / H=4.5$. Further downstream, $x / H>6$, the flow recovers towards a fully turbulent, attached, boundary layer.

Comparisons between simulations and experimental data are presented in figures $7-10$, while further statistical properties derived from the simulations are given 

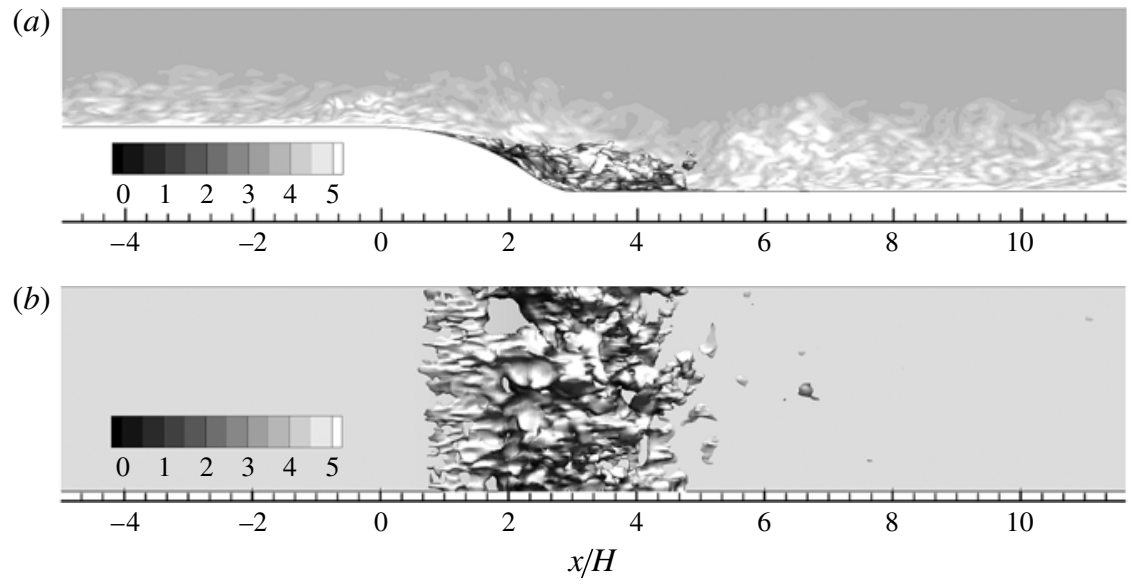

FIGURE 6. Baseline flow: instantaneous-flow visualisation with vorticity magnitude contours (in terms of multiples of $U_{i n} / H$ ) and isosurface of $u=0$. (a) Side view; (b) top view.

in figures 11 and 12. To illustrate sensitivity of the solutions to mesh disposition and SGS modelling, figures 7-9 include results from both LES simulations for the baseline case, with differences between them explained in the previous section.

The streamwise variation of the pressure coefficient $C_{p}$ and the skin-friction coefficient $C_{f}$ are shown in figures 7(a) and 7(b), respectively. Results are included for all three grids examined, and differences are seen to be quite modest and confined mainly to the post-reattachment region. The time-averaged separation occurs at $x / H=0.85$, in both simulations and in the experiment, and the flow reattaches at $x / H=4.15$. As is common in separated near-wall flows, the wall-pressure distribution features a plateau within this region, although its length is short, consistent with the rather thin, elongated tongue and tail of recirculating flow upstream of $x / H=2$ and downstream of $x / H=3.5$. The $C_{f}$ variation shows that the near-wall flow accelerates well upstream of the curved section as a consequence of the pressure drop induced by the convex curvature further downstream, reflecting the elliptic nature of the pressure field. While the computed nearwall acceleration appears in excess of that measured, as implied by the single experimental value of $C_{f}$ at $x / H=0$ (deduced from the wall-nearest velocity data in both experiment and simulation), it is curious - if not inconsistent - that the experimental pressure trough is deeper than that predicted, regardless of mesh refinement, suggesting a more intense acceleration in the experiment. However, it must be borne in mind that $C_{f}$ is indicative primarily of the response of the layer very close to the wall, while the wall pressure reflects the global response of the entire boundary layer when subjected to the effects of curvature. Thus, the differences observed in figure 7 might be assumed to indicate some differences in the structure of the predicted and measured boundary layers as the separation region is approached. While there are no experimental boundary-layer data that would allow this inference to be tested upstream of $x / H=0$, data beyond that position do not provide any indications of defects in the predicted flow field. Nor are there any indications that an inconsistent normalisation of $C_{p}$ or a misrepresentation of the boundary layer on the upper wall is responsible. It is arguably relevant in the present context to draw attention to a recent highly resolved LES study by Garcia-Villalba et al. (2009) of a flow around a three-dimensional hill. That study also featured significant discrepancies in $C_{p}$ as the flow approached the hill crest and progressed towards separation in the leeward side, 

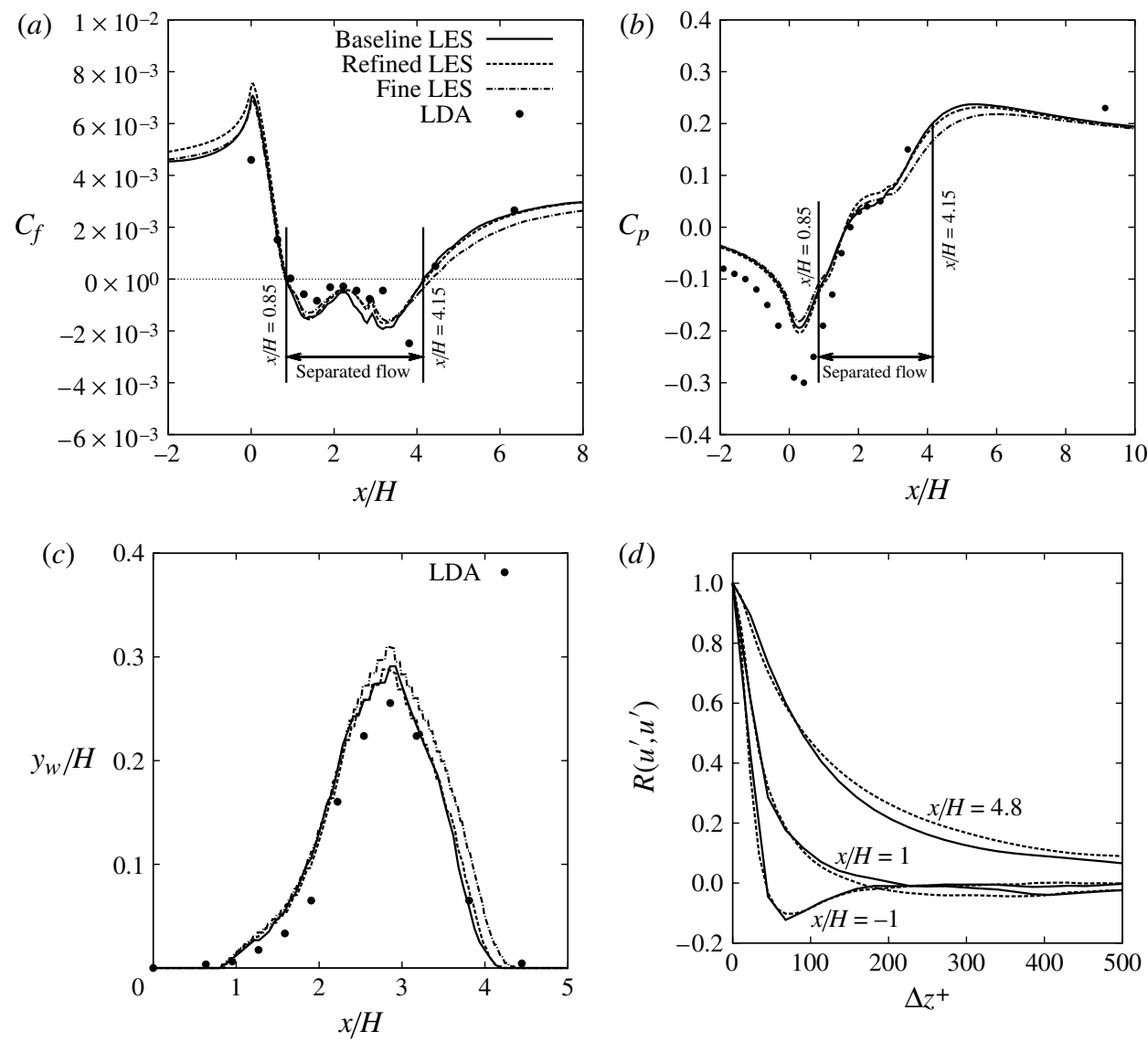

FIGURE 7. Baseline flow: (a) spanwise-averaged streamwise variation of the skin-friction coefficient $C_{f} ;(b)$ spanwise-averaged streamwise variation of the pressure coefficient $C_{p} ;(c)$ spanwise-averaged streamwise variation of height of reverse-flow layer above the ramp wall; (d) spanwise variation of two-point correlation function of the streamwise fluctuations. Lines and symbols: •, experimental results of Zhang \& Zhong (2010); - , LES results, baseline mesh; ---, LES results, refined mesh; ---, LES results, fine mesh.

despite a correct prediction of the boundary-layer structure. In that study too, resolution was demonstrably not the issue. While the two flows contrasted here differ significantly, the comparison serves to illustrate the particular sensitivity of the wall pressure in flow separating from gently curving surfaces, especially if the separation region is relatively thin. Pleasingly, once separation has occurred, the pressure rise towards the plateau, the plateau itself and the subsequent rise towards reattachment are correctly captured. It is finally noted that the uneven variation of $C_{f}$ within the recirculation zone reflects the lack of curvature continuity in the shape of the wall, as expressed by $(2.1 b)$.

A good global indicator of the quality with which the separation process as a whole is predicted is given by the locus of zero streamwise velocity within the recirculation bubble. This is shown in figure $7(c)$. As is seen, separation is predicted at the correct position and varies in close accord with the experimental data up to around $x / H=4$. While the recirculation region is $3.3 \mathrm{H}$ long, it is only about $0.6 \mathrm{H}$ high (about twice the thickness of the reverse-flow layer) - hence, rather thin, as already noted. The zero-velocity locus 

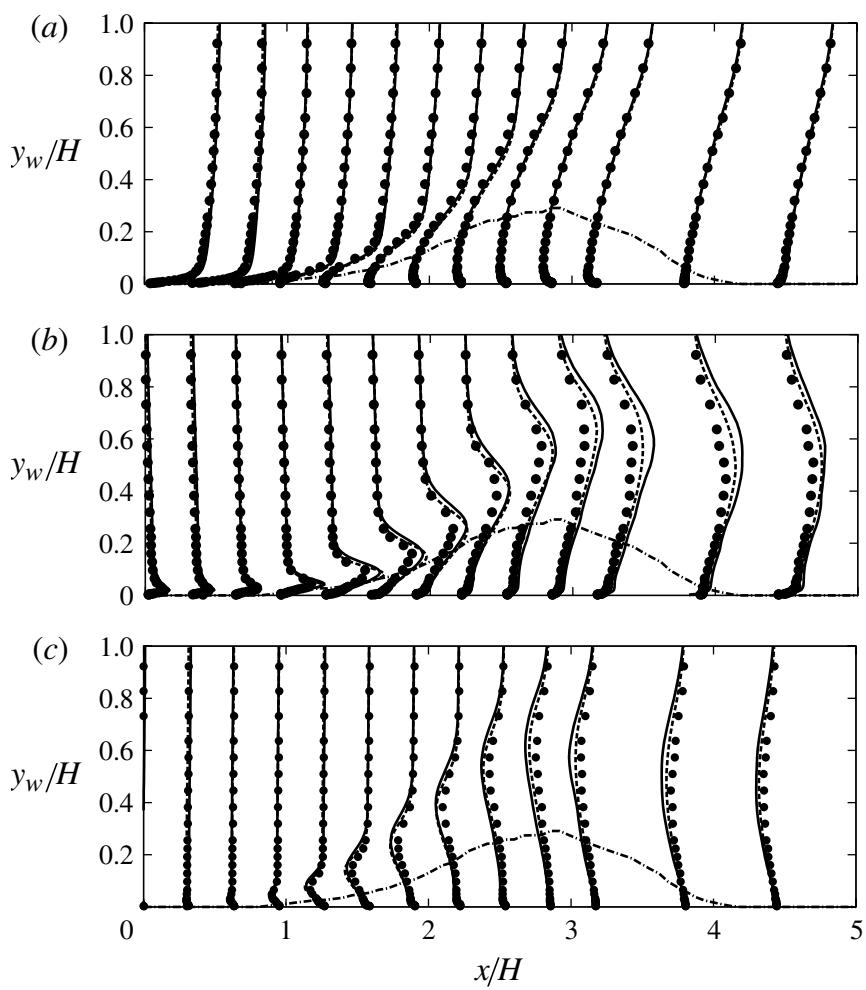

FIGURE 8. Baseline-flow, spanwise-averaged properties: (a) streamwise velocity $U / U_{i n}$, (b) streamwise Reynolds stress $\overline{u u} / U_{i n}^{2}$; (c) shear stress $\overline{u v} / U_{i n}^{2}$. The LES-derived zerostreamwise-velocity locus is shown as a dash-dot line; •, experimental results of Zhang \& Zhong (2010); - , LES results, baseline mesh; ---, LES results, refined mesh.

indicates an especially thin and elongated reverse-flow zone following separation, up to $x / H=1.8$. Interestingly, this is a feature also observed in Garcia-Villalba et al. (2009), and is associated with the spatially and temporally varying separation process, with the flow being intermittently attached and separated around the time-averaged separation location.

Attention has already been drawn to figure $7(d)$, in relation to the streak distance in the upstream boundary layer. Its inclusion is intended, primarily, to reinforce the observation derived from all other figures that the results of the simulations are only marginally sensitive to the grid and the SGS model. Moreover, the variations of $R_{u u}$ demonstrate that the spanwise width of the domain is sufficient to secure spanwise decorrelation. Increasing the spanwise domain further would have been possible, in principle, but would have necessitated the inclusion of a third jet, with its cavity, thus substantially increasing the resource requirements.

Comprehensive comparisons of predicted streamwise-velocity and streamwiseReynolds-stress profiles with all available experimental data are given in figure 8 , while magnified views of profiles of $U, \overline{u u}$ and $\overline{u v}$, at four different positions close to the separation and reattachment points are given in figure 9. The predicted zero-velocity locus, given earlier in figure 7(c), is included again in figure 8 for reference. Agreement with experiment is generally close. The only significant difference is that the turbulence activity in the outer part of the separated shear layer is modestly overestimated by the LES. As anticipated, there is a strong rise of the streamwise fluctuations once the flow 


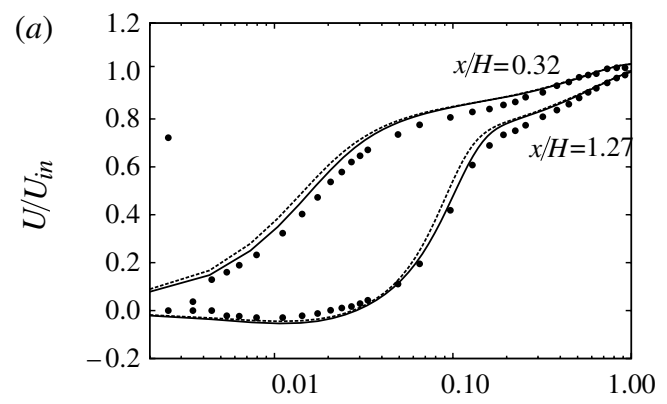

(b)
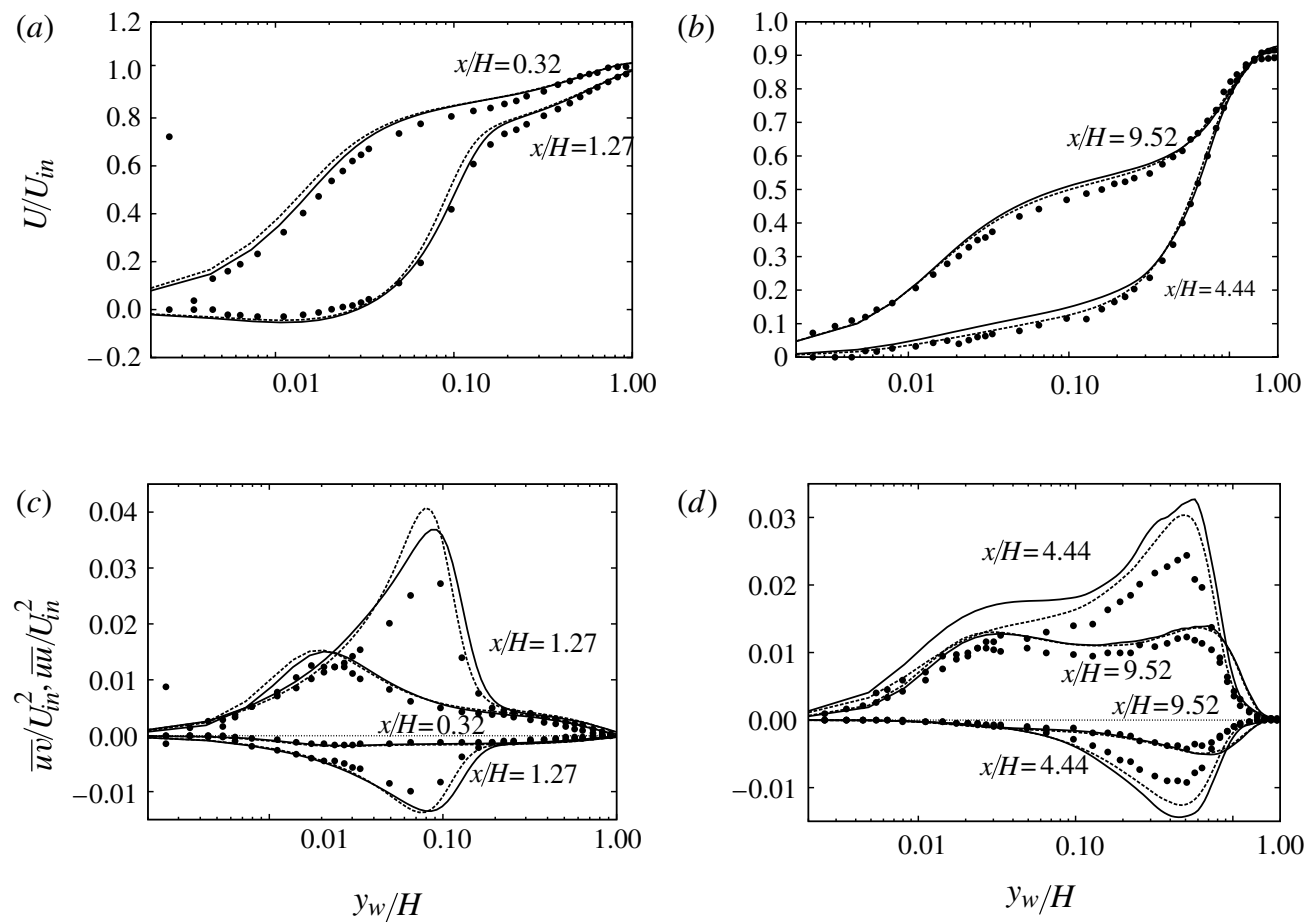

FIGURE 9. Baseline-flow, spanwise-averaged properties, selected profiles: $(a, b)$ streamwise velocity; $(c, d)$ streamwise and shear Reynolds stress; - , LES results, baseline mesh; -- -, LES results, refined mesh.

separates. In fact, this rise begins well ahead of the separation location, as is brought out clearly in figure $9(c)$, which indicates very high values of the ratio $\overline{u u} / \overline{u v}$ at $x / H=0.32$. A probability density function (p.d.f.) of the separation location, given in figure 10, shows separation not to occur further upstream than $x / H=0.5$, with a few rare exceptions (cf. figure 6). Hence, the high value in streamwise stress at $x / H=0.32$ is taken as indicative of the destabilising influence of the adverse pressure gradient that sets in at around $x / H=0.2$. Following separation, the increase in the streamwise stress is accompanied by a corresponding increase in shear stress, although the ratio $\overline{u u} / \overline{u v}$ suggests a turbulence structure in the separated shear layer that is far from equilibrium - an issue that will be revisited later by reference to a few turbulence-energy budgets. The reverse flow in the recirculation region, which is usually quite sensitive to resolution and difficult to capture accurately in LES, is remarkably well reproduced, and reaches a minimum of $U_{\min } / U_{\text {in }}=-0.1$ around $x / H=2.70$, in close accord with the experimental value.

Turbulence-energy budgets at three locations, spanning the separation-to-reattachment range, are shown in figure 11. All contributions to the budget are scaled with the freestream inlet velocity and the step height. The viscous-diffusion terms are negligible, except very close to the wall, and have thus been omitted from the plots. All three budgets share common features, the principal difference lying in the magnitude of the contributions. Close to the separation point $(x / H=0.95)$, production rises quickly to a very high level, relative to that in the upstream boundary layer, reaching a maximum at around $x / H=1.3$. This is balanced by dissipation, pressure diffusion and turbulent 


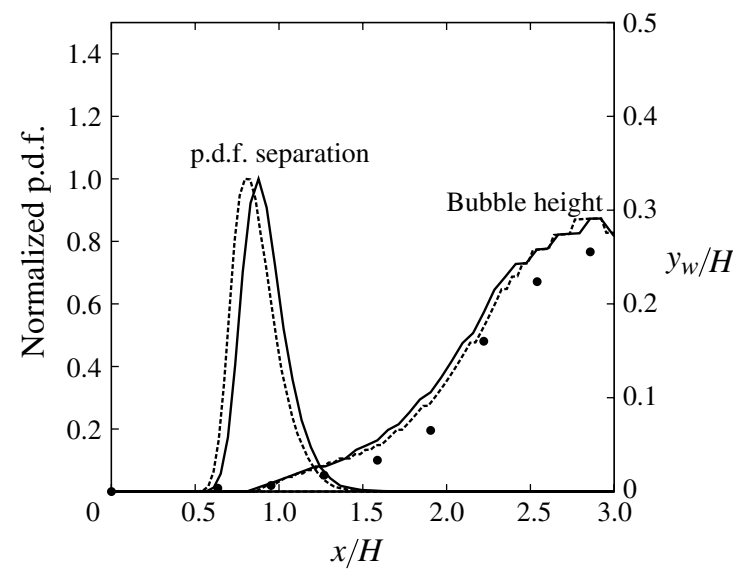

FIGURE 10. Baseline flow: p.d.f. of the position of the upstream-most separation point sampled across the span; - , LES results, baseline mesh; ---, LES results, refined mesh.
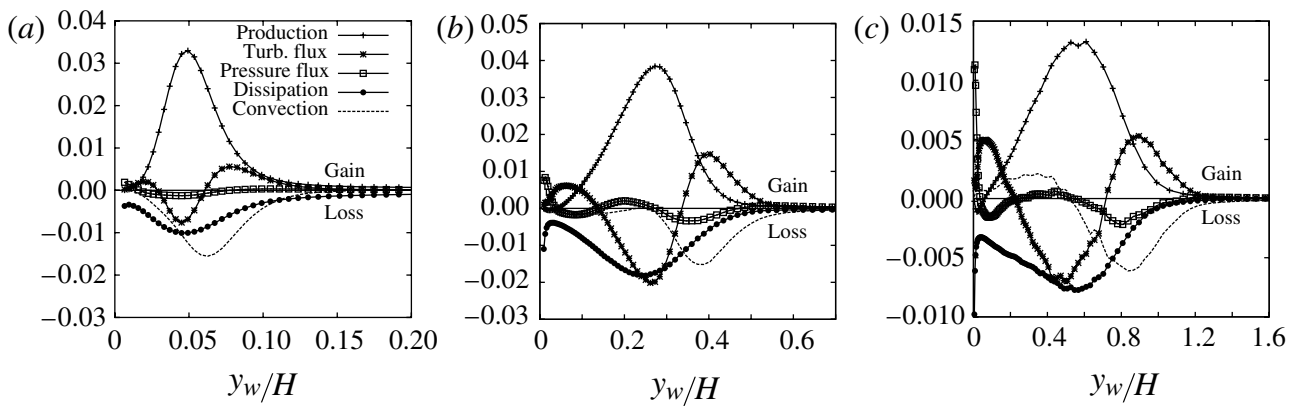

FIGURE 11. Baseline flow: spanwise-averaged turbulence-energy budgets; $(a) x / H=0.95$, (b) $x / H=1.90$ and $(c) x / H=3.80$.

transport, the first being much lower than production, however, especially in the early stages following separation. Turbulent transport is a very important contributor, allowing turbulence energy to diffuse towards the wall and away into the outer region, in an effort to compensate for the difference between the high level of production and low level of dissipation in the central region of the separated shear layer. The strong imbalance between production and dissipation, the former exceeding the latter by a factor 3 close to the separation point, thus highlights the fact that the flow is very far from a state of equilibrium. This also applies to other streamwise locations in the separated shear layer, albeit to a lesser extent. Thus, at $x / H=1.9$, which is almost in the middle of the recirculation zone, production exceeds dissipation by a more modest factor 2 . Diffusion and convection, the latter confined mainly to the outer portion of the shear layer, make important contributions to the balance. In common with other streamwise locations, the balance in the outer region at $x / H=1.9$ is characterised by dissipative and convective losses being counteracted by moderate production and positive turbulent diffusion. Finally, close to the reattachment point, the main difference relative to the other two locations is that the production has declined to around $20 \%$ of its maximum value, due to relatively low shear straining, with other contributions following suit. In most other respects, this budget has features similar to those at other locations. 

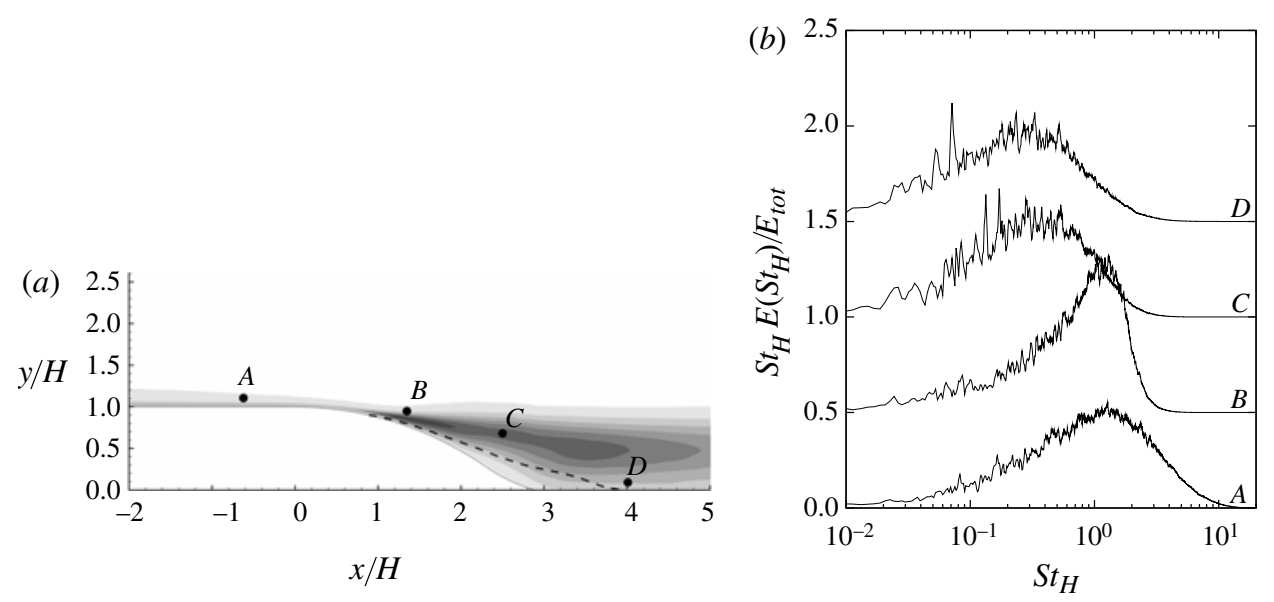

FIgURE 12. Baseline flow: pre-multiplied energy spectra. (a) Positions of the four probes at which samples for energy spectra were taken; $(b)$ energy spectra.

There has been a significant amount of discussion in the literature about instability modes in separation bubbles. Three modes are said to be pertinent: the shear-layer mode, the shedding (or step) mode and the flapping mode. The former is associated with the Kelvin-Helmholtz $(\mathrm{K}-\mathrm{H})$ waves, depends only on the state of the boundary layer around the point of separation, and is associated with the much studied instability caused by the inflectional profile in the shear layer. Hasan \& Khan (1992) have reported that the Strouhal number, based on the momentum thickness at the point of separation, is $S t_{\theta}=0.012$. The second mode appears to scale with the height of the step from which the flow separates, or the length of the shear layer. This mode is characterised by $S t_{H}=0.2$ and is said to be linked to the shear-layer mode by a sequence of vortex pairing as the shear layer evolves (Hasan \& Khan 1992). This mode has been singled out as offering the potential of optimum control by pulsed perturbations through resonance between the actuation frequency and the shedding mode. The third, flapping, mode is associated with the interaction - a temporal imbalance - between the entrainment of fluid into the separation region and the injection of fluid from the separation point into the separated bubble (hence directly related to the size of the separation bubble), and is generally observed to be much lower than the shedding frequency - reported by Dandois et al. (2007) to be of the order $S t_{L}=0.12-0.18$, where $L$ is the average recirculation-bubble length.

In the present case, $S t_{H}=0.2$ corresponds to $S t_{\theta}=0.011-$ i.e. virtually identical to the shear-layer mode - because at the nominal separation location, the present simulations for the baseline case yield $\theta / H=0.055$ (see figure 19 discussed in the next section). To investigate the existence of the above instability modes, in the present case Hanningwindowed energy spectra (Oppenheim \& Schafer 2009) have been obtained for the baseline simulation on the 'refined mesh'. Four such spectra are shown in figure 12, derived at locations A-D, which are identified in figure 12(a) (the dashed line is the zero-streamwise-velocity locus). Upstream of the separation, the energy peaks occur at high-frequency values, around $S t_{H}=1-2$. In contrast, at locations $C$ and $D$, the peak shifts markedly to around $S t_{H}=0.2$. There is no clear evidence of another distinct mode, except for a hint to an elevated spectral region around $S t_{H}=0.08$, which is close to the first subharmonic of the $\mathrm{K}-\mathrm{H}$ mode, possibly indicative of vortex pairing. Thus, to some extent, 
(a)

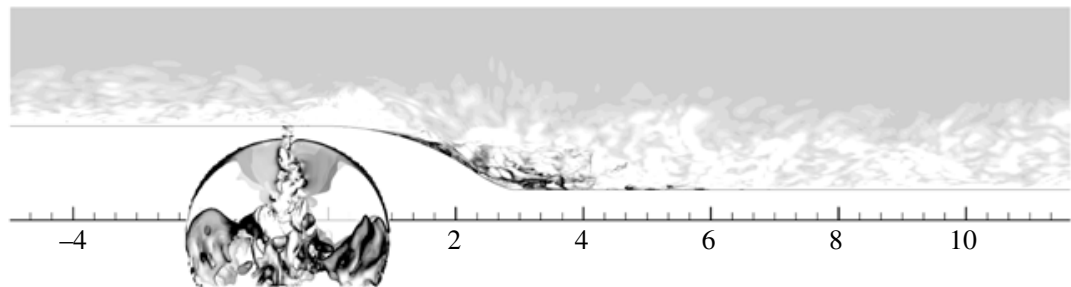

(b)

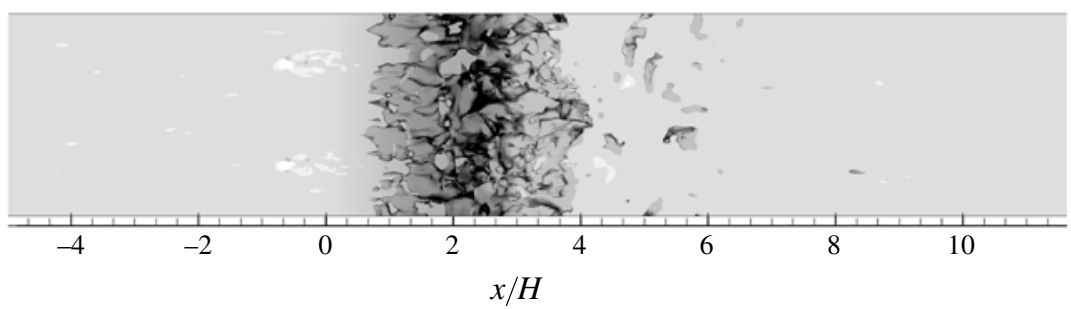

FIGURE 13. Reference actuated case $C_{\text {ref }}$ : instantaneous flow at maximum ejection phase visualised with vorticity magnitude contours and zero-velocity iso-surface. (a) Side view; (b) top view (note bright regions downstream and on either side of two orifices, indicating elevated vorticity magnitude).

the spectra support the choice of $S t_{H}=0.2$ as the actuation frequency for which results follow.

\subsection{Synthetic-jet actuation: reference case}

In what follows, a discussion is provided of the effects of synthetic-jet actuation on the baseline flow discussed above. The present section confines itself to a description of the reference-injection case $C_{\text {ref }}$ (see table 1), for which experimental data are available, while subsequent sections deal with the limited parametric exploration in which the injection velocity and angle are varied.

Figure 13 shows an instantaneous image, corresponding to that of figure 6 for the baseline case, at an actuation phase at which the injection rate is close to its maximum value. The cavity has been deliberately included to demonstrate that the actuation actually results in two synthetic jets, one in the boundary layer and the other within the cavity. Hence, the fluid ejected into the boundary layer is, in general, significantly perturbed, having a highly non-uniform velocity profile and carrying with it the turbulence contained in the cavity. The ejection involves the expulsion of vorticity, visible in figure 13 as weak bright streaks on either side and downstream of the jet orifices (top view), and also ahead of the curved ramp in the jet centre-plane (side view). The visualisation suggests, albeit tentatively, that the effect of the jets on the size of the separation zone is rather modest: a local delay in the separation, represented by a downstream shift of around $0.5 \mathrm{H}$ across a spanwise slab of roughly $2 d$. Hence, a first indication emerging from figure 13 is that the modifications induced by the round jets are substantially weaker and more subtle than those produced by slot jets, where reductions typically of order $30 \%$ in the size of recirculation zone across their entire span are achieved. The more subtle effect in the present configuration is evidently due to the large spanwise jet-to-jet separation (10d). This large separation distance also means that the jets do not interact, except perhaps following separation when the largest turbulent scale is of the order of the jet-separation distance. Similar observations also apply to isolated solid vortex generators. 

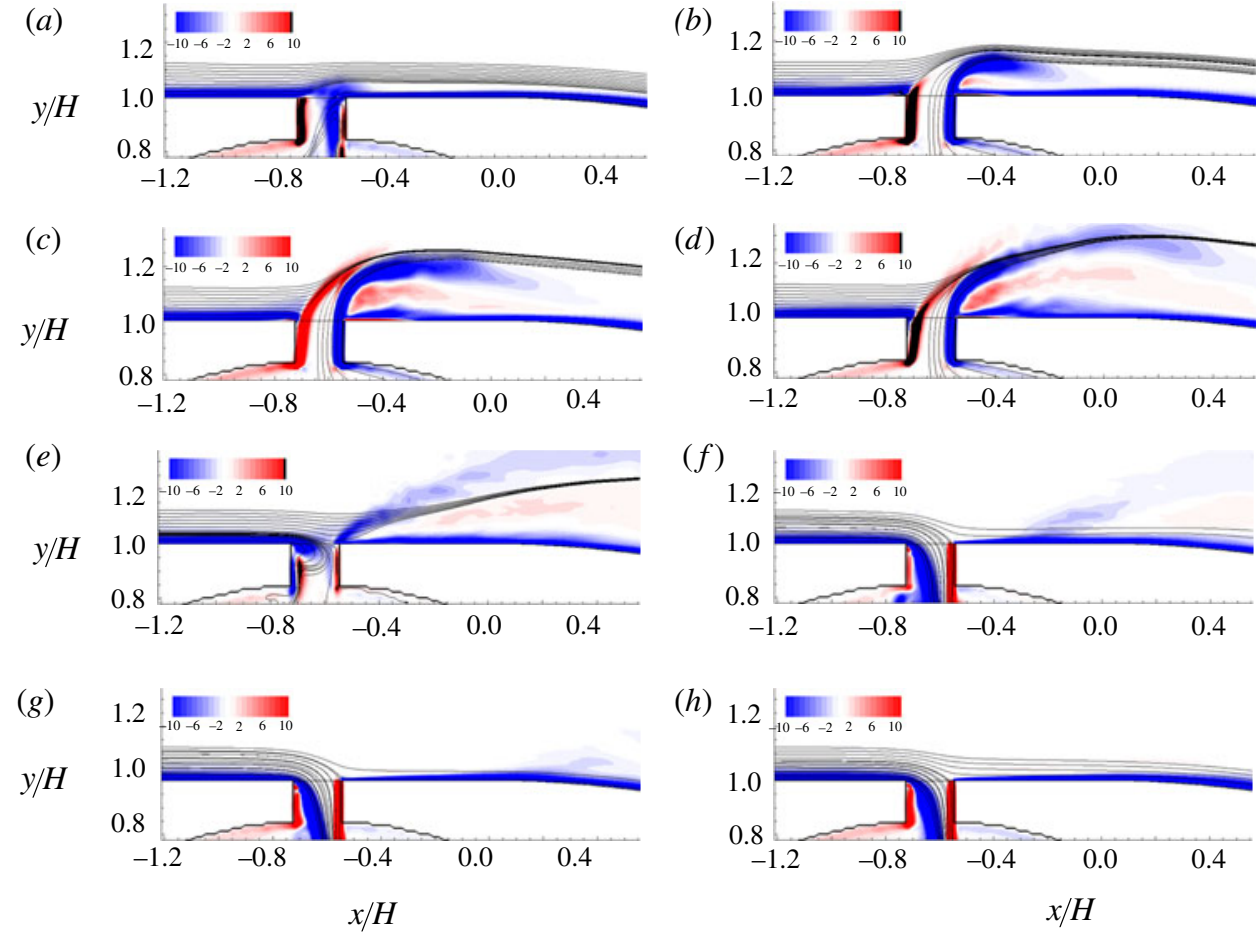

FIGURE 14. Reference actuated case $C_{r e f}$ : phase-averaged streamlines and isocontours of spanwise vorticity, $\left\langle\omega_{z}\right\rangle$, for 8 regularly spaced phases spanning the full injection cycle across the jet mid-plane. $(a-d)$ Ejection phase; $(e-h)$ suction phase. $(a) 0,(b) \pi / 4,(c) 4 \pi / 8$, (d) $6 \pi / 8,(e) 8 \pi / 8,(f) 10 \pi / 8,(g) 12 \pi / 8$ and $(h) 14 \pi / 8$.

The details of the actuation process around and within the orifice are shown in figure 14 by way of 8 phase-averaged plots of the spanwise vorticity component $\left\langle\omega_{z}\right\rangle$ on the centreplane, covering the entire actuation cycle. These are best viewed in conjunction with the phase-averaged pressure-Laplacian contours in figures 1 and 2, showing the evolution of the vortical structures in the same region. During the outstroke phase (figure $14 a-d$ ) positive vorticity within the shear layer of the jet emanating from the upstream lip of the nozzle is shed into the upper portion of the boundary layer. At the same time, negative vorticity is shed by the leeward shear layer into the region behind the orifice. These two regions are, in effect, cross-sections through the vortex ring seen in figure 1 . Between the lower (negative-vorticity) layer of the jet and the wall nestles a region of weak positive vorticity, indicating the presence of a secondary vortex induced by the primary vortex ring, and hence a reverse-flow region. Below that region, however, very close to the wall, the vorticity is again negative, presumably due to the spanwise transport of negative-vorticity fluid by transverse motion from outer regions towards the centre-plane. During the instroke phase (figure $14 e-h$ ), the synthetic jet acts as a sink flow. The fluid drawn into the orifice at its upstream edge separates within the orifice, and boundary-layer fluid with negative vorticity is strongly drawn into the cavity for the entire duration of the suction phase, accelerating and thinning the boundary layer and giving rise to a negative value of the timeaveraged velocity in the upstream portion of the orifice. At the downstream lip, suction also leads to a downward motion, with positive vorticity at the orifice wall. However, here, the fluid ingested originates from the rear, decelerating boundary layer, which contains 


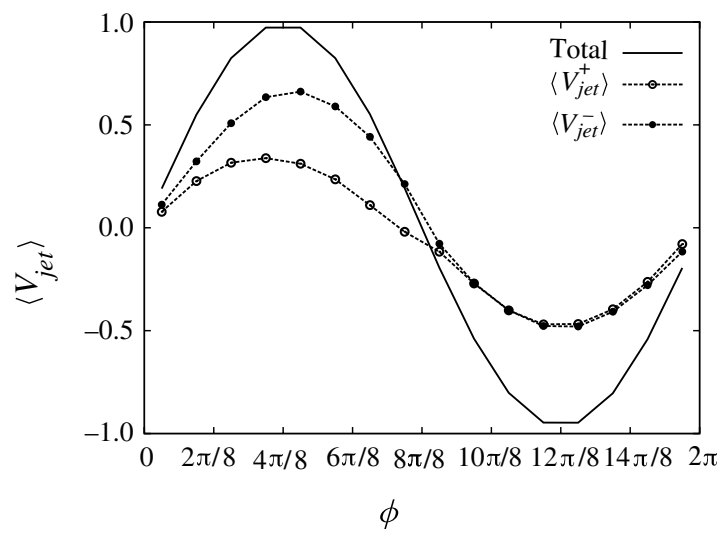

FIGURE 15. Reference actuated case $C_{r e f}$ : phase-wise $(\phi)$ variations of area-averaged contributions of phase-averaged jet velocity over the upstream $(+)$ and downstream (-) halves of the orifice and their sum (i.e. the orifice-averaged velocity).

predominantly negative vorticity, an interaction which opposes the ingestion process. Thus, at the time-averaged level, the jet velocity is positive in this region.

An important consequence of the highly asymmetric interaction between the boundary layer and the injection process is that the phase- and time-averaged flow and vorticity distribution vary greatly across the orifice. This is exemplified by figure 15, which shows separate contributions of the area-averaged jet velocity over the upstream and downstream halves of the orifice area (denoted by the superscripts ' + ' and '- ', respectively) during the actuation cycle, with their sum being the total sinusoidal variation that must comply with the cycle-averaged zero-mass-flux constraint. As is seen, the ejection velocity over the front half of the orifice is much lower than that over the rear half, and this indicates, globally, the strong distortions arising from the collision of the boundary layer with the ejected jet fluid. Clearly, the time-integral of the upstream contribution is negative, while that downstream is positive. Hence, the time-averaged flow at the upstream portion of the orifice is into the cavity, which is very different from the conditions in continuous injection. The implication is thus that ignoring this variation in any computational simulation - say, by prescribing the injection conditions as a temporally varying plug flow - is likely to have a significant negative impact on the realism of the simulation. It is this fact that primarily motivates the inclusion of the cavity in the simulation.

Figure 16 is the first of several similar plots, included for different actuation scenarios, that attempt to bring out some major differences in respect of the periodic motions induced by the actuation. The figure shows, for 8 equally spaced phases, iso-surfaces of $0.1 U_{\text {in }}$ and $-0.1 U_{\text {in }}$ in the streamwise and wall-normal velocity components. The plots cover a streamwise distance of about 6 orifice diameters, within which the periodic velocity components are especially pronounced.

At the start of the ejection, $\phi=0$, the periodic fluctuation downstream of the orifice is predominantly positive. This reflects the fact that the suction preceding this phase $(\phi=$ $2 \pi-\pi / 8$ ), coupled with a predominantly negative wall-normal motion, draws down highvelocity fluid from upper layers towards the wall, leading to a streamwise acceleration (positive increment). As the ejection progresses, the wall-normal motion is predominantly positive, except on the spanwise edges of this positive-velocity region (see especially lower plot at $4 \pi / 8$ ). This ejection then induces a deceleration, away from the wall, reflecting the blockage posed by the ejected fluid to the oncoming flow. Simultaneously, there also arises 

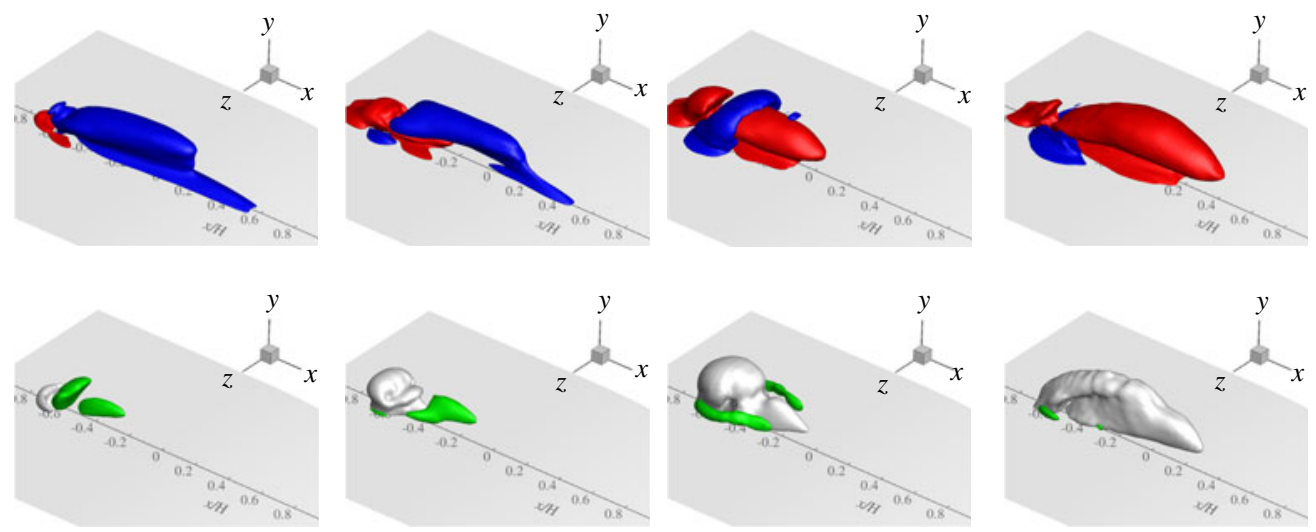

0

$2 \pi / 8$

$4 \pi / 8$

$6 \pi / 8$
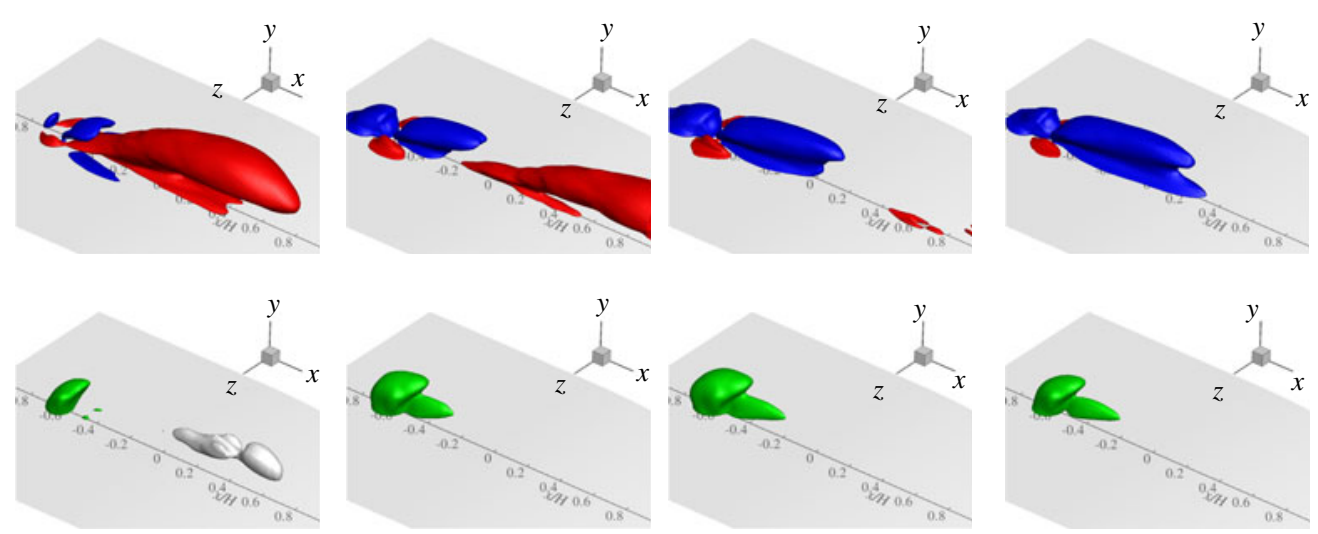

$8 \pi / 8$

$10 \pi / 8$

$12 \pi / 8$

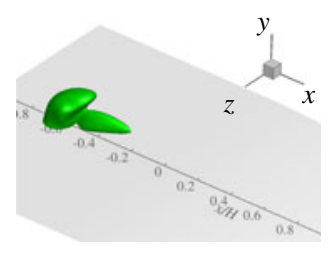

$14 \pi / 8$

FIgURE 16. Baseline case $C_{r e f}$ : iso-surfaces of $\tilde{u}= \pm 0.1 U_{\text {in }}(+0.1$ in blue and -0.1 in red), and of $\tilde{v}= \pm 0.1 U_{\text {in }}$ ( +0.1 in grey and -0.1 in green), at 8 equally spaced phase values (progressing left to right and top to bottom) covering a whole cycle.

a second region of deceleration, close to the wall, as a consequence of the shielding effect of the jet. This creates a low-pressure region behind the jet into which low-momentum near-wall fluid is drawn from outer spanwise regions towards the centre-plane. The adverse pressure gradient acting on this low-momentum fluid then induces a reverse flow over some portions of the ejection phase (figure 14). Towards the end of ejection, the periodic field is thus dominated by an upward wall-normal motion and a decelerated streamwisevelocity region extending to around $5 d$. Importantly, the spanwise extent of this region is rather narrow - only around $2 d$. During the suction phase, the wall-normal velocity around the orifice is negative, and this induces the transport of high-momentum fluid from outer regions towards the wall (figure 14), resulting in a positive streamwise velocity fluctuation. The sequence of negative and positive fluctuations is seen especially well in the upper plot at $\phi=10 \pi / 8$. Towards the end of the suction phase, $\phi=14 \pi / 8$, the flow has almost returned to the state $\phi=0$ at the top left-hand side of the figure.

An important aspect of the streamwise fluctuations in figure 16 is that the near-wall region is affected, primarily, by positive fluctuations, while negative fluctuations are 

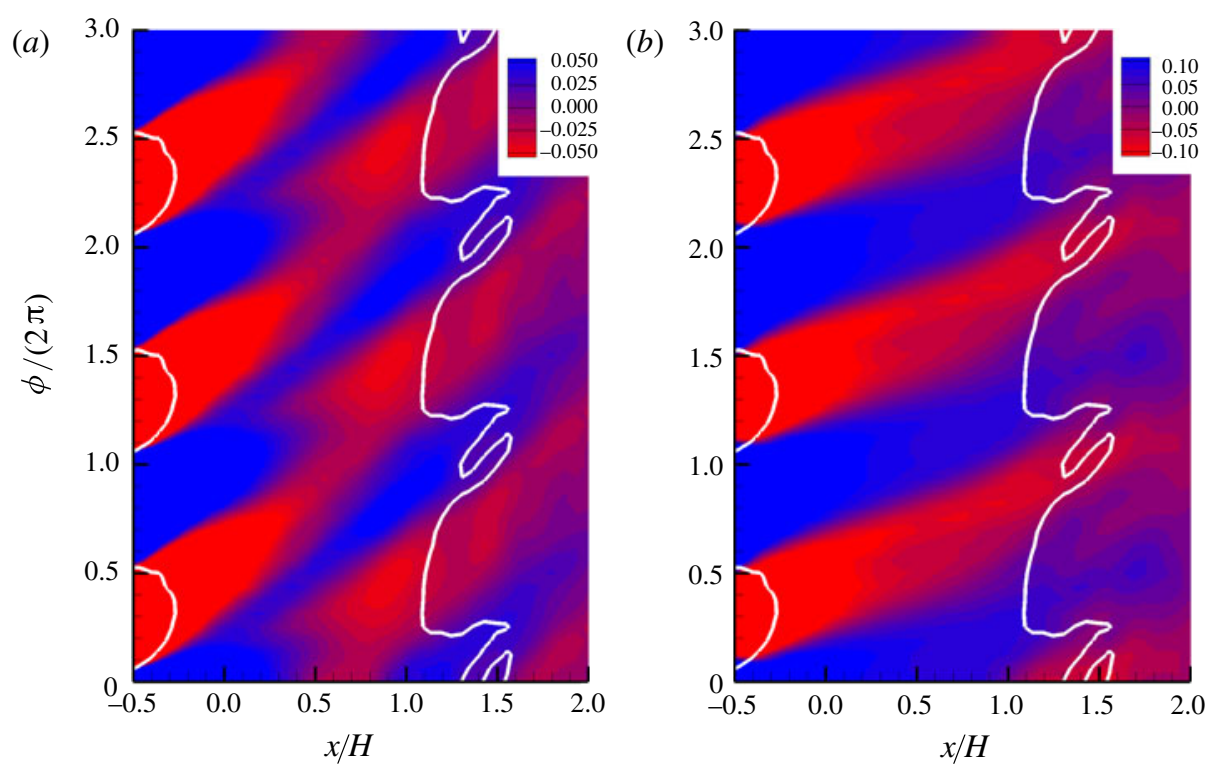

FIGURE 17. Reference actuated case $C_{\text {ref }}$ : space-time plots of periodic streamwise velocity fluctuation $\tilde{u} / U_{i n}$ in the jet centre-plane for $(a) y_{w} / H=0.01$ and $(b) y_{w} / H=0.085$. The white line indicates the separation point on the jet centre-line (derived from the reversal of wall-nearest velocity).

predominant in outer regions. Because positive fluctuations at the wall tend to push the separation location in the positive streamwise direction, the consequence of the above is that, on average, the periodic component is expected to produce a downstream shift of the separation line around the jet centre-line. This is brought out in figure 17, which shows space-time maps of streamwise periodic-velocity fluctuations over three actuation cycles at two wall-normal elevations, $y_{w} / H=0.01$ and 0.085 , respectively. The maps include the phase-average variation of the separation point on the orifice centre-line. It is recalled that the separation point in the baseline flow is at $x / H=0.85$, while the time-average value for the present reference actuated case is $x / H=1.18$. As is seen from figure 16, the phaseaverage value varies between 1.0 and 1.5 , but the more important point to highlight is that separation is delayed primarily by positive streamwise-velocity fluctuation at the wall (i.e. at $\left.y_{w} / H=0.01\right)$. It is also interesting to note that, away from the wall $\left(y_{w} / H=0.085\right)$, the characteristic line identifying the progression of perturbations has a much shallower angle, signifying a higher convection velocity with which the separation delay is not correlated. The conditions at this wall-normal distance correspond to the outer tongue-like structures seen in figure 16.

As will be shown below, the strength of the periodic component declines substantially as the separation region is approached. However, the above predominance of positive nearwall perturbation is still likely to contribute to the delay of separation, enhancing any actuation-induced stochastic turbulence and mixing by transverse circulation.

Figure 18 shows the time-averaged zero-streamwise-velocity locus, the spanwise location of the separation line and the separation-bubble length for the reference actuated case, relative to the baseline flow. The injection is seen to lead to a reduction of the length and height of the separation bubble, with the maximum reduction of the former being around $17 \%$ at the jet centre-plane. This level is considerably lower than that obtained with slot jets, for reasons discussed already. It is also observed that the spanwise region of 

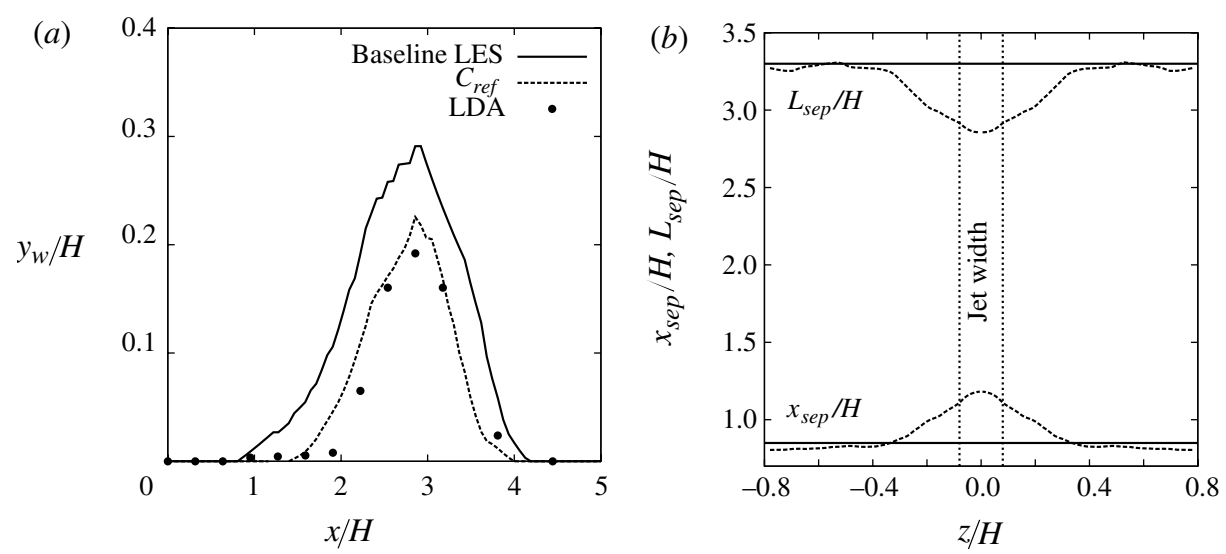

FIGURE 18. Reference actuated case $C_{r e f}$ : $(a)$ height of reverse-flow layer in jet centre-plane and $(b)$ locus of the separation $x_{\text {sep }}$ and separation length $L_{\text {sep }}$. Lines: - , baseline case; - - , actuated case.

influence extends only to around $1 d$ on either side of the orifice, suggesting that a densely packed row of jets is required if an effect is to be achieved that goes beyond the action of a single jet that is divorced from its neighbours. Agreement of the zero-velocity locus with experiment is fairly close, except in the upstream portion of the recirculation zone where the separated region is very thin and difficult to map accurately.

The global effect of the actuation on the structure of the boundary layer approaching the separation point on the centre-plane is illustrated in figure 19 by way of the streamwise variation of the momentum thickness $\theta / H$. These were derived in accordance with the expression by Dandois et al. (2007):

$$
\theta=\int_{y_{\min }}^{+\infty} \frac{U-U_{\min }}{U_{\text {in }}-U_{\min }}\left(1-\frac{U-U_{\min }}{U_{\text {in }}-U_{\min }}\right) \mathrm{d} y .
$$

Figure 19 also contains results derived from the parametric exploration that is discussed in the following two sections; comments here relate only to the comparison of the reference actuated flow to the baseline flow. The relevance of including $\theta$ is that it represents the jet-induced boundary-layer thickening as a consequence of obstruction and adverse pressure gradient in the near-field wake of the jet. It is appropriate to note here that momentum thickening is not, in itself, necessarily desirable, because such thickening indicates deceleration on the lower region of the boundary layer, thus potentially reducing the boundary layer's ability to resist separation. However, the actuation also results in momentum mixing and enhanced turbulence further downstream, and the net result turns out to be a desirable delay in the separation. Thus, it can be argued that the optimum combination is low jet-induced momentum thickening coupled with intensive downstream mixing.

Figure 19 shows, first, that the general trend of $\theta$ is to decline ahead of the orifice, due to ramp-associated acceleration. In the presence of actuation, this acceleration is enhanced by the fact that the time-averaged flow in the upstream portion in the orifice is directed into the cavity (constituting suction), so that the boundary layer is pulled towards the orifice. Further downstream, beyond $x / H=0.1-0.2$, the flow is subjected, overall, to an adverse pressure gradient as the boundary layer progresses towards separation, hence provoking a rapid rise in the momentum thickness. The introduction of actuation causes a significant 


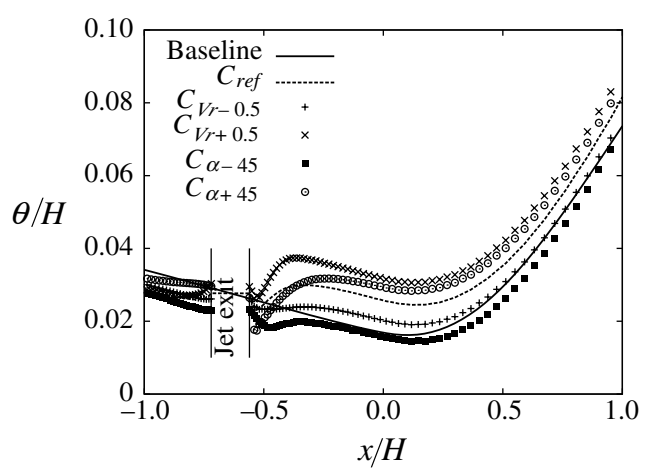

FIGURE 19. All flow cases examined: streamwise variations of momentum thickness $\theta$ along the jet centre-plane.

rise of the momentum thickness behind the jet as a consequence of the blockage of the jet and loss of momentum. Further downstream, beyond about two orifice diameters, the variation of the momentum thickness, although elevated, is broadly similar to that of the baseline boundary layer. However, the enhanced mixing that the actuation causes tends to partially reverse the elevation of the momentum thickness, the difference relative to the baseline level being modest by the time the boundary layer reaches the separation region. This mixing is illustrated by the downwards-directed contours of streamwise velocity downstream of the jet in figure 3 .

An extensive comparison between measured and computed profiles of time-mean streamwise velocity, streamwise normal stress and shear stress on the jet centre-plane is given in figure 20. Again, agreement is close in most regions, the exception being in respect of the turbulence activity in the outer part of the flow above the separated region. The effect of the injection is most pronounced in the three or four upstream-most profiles of mean velocity and streamwise turbulence intensity. The former point to a deceleration within the layer $y_{w} / H=0.3$, into which jet fluid is ejected, and a thickening of the boundary layer as a whole, with inflection points between the inner and outer layers. This deceleration is consistent with the predominance of negative periodic fluctuations in the outer region, identified earlier by reference to figure 16 . The outer peaks in streamwise stress indicate a substantially enhanced level of unsteadiness in and below the inflection layer, but the effect of the shear stress is relatively modest, suggesting that the peaks are primarily due to the periodic component, which does not cause a significant level of momentum mixing. As will also be shown below, the fairly fast decay in the turbulenceintensity peaks in the streamwise direction is due mainly to a correspondingly rapid decline in the strength of the coherent component, which dominates the total intensity immediately downstream of the injection. This component is only a modest proportion of the total unsteadiness by the time the flow reaches the separation zone, so that the increased resistance to separation is due, primarily, to enhanced stochastic turbulence and mixing by transverse circulation created by the injection of vorticity into the boundary layer. These observations suggest that the level of separation control effected by the jet injection suffers from the actuation being too far upstream of the separation location and insufficiently targeted towards the agitation of the near-wall layer.

The periodic and stochastic contributions to the total time-averaged turbulent stresses may be determined via the triple decomposition defined by (3.1). This is done in figure 21 for the streamwise normal stress (upper part of each figure) and shear stress (lower part 

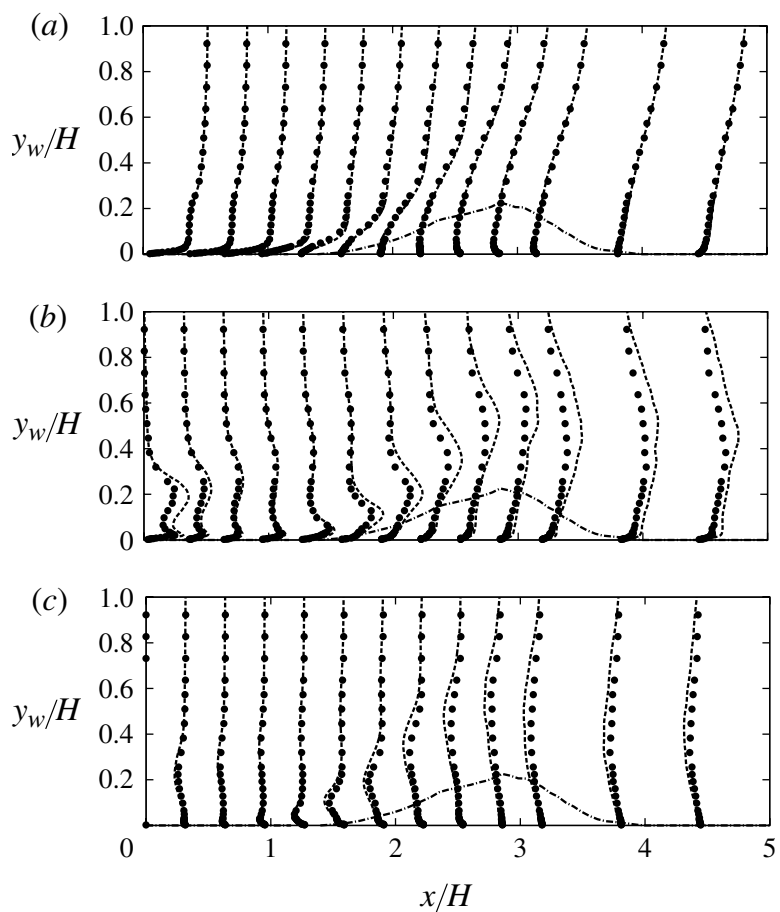

FIGURE 20. Reference actuated case $C_{r e f}$, flow properties in jet centre-plane: $(a)$ streamwise velocity $U ;(b)$ streamwise Reynolds stress $\overline{u u} / U_{i n}^{2} ;(c)$ shear stress $\overline{u v} / U_{i n}^{2}$. The LES-derived locus of zero-streamwise velocity is shown as a dash-dot line; •, experimental results of Zhang \& Zhong (2010); ---, LES results.

of each figure) at four streamwise locations, selected from the 13 included in figure 20. Each plot contains four distributions per stress component, representing respectively: the total value with actuation $\overline{u_{i} u_{j}}$, the periodic contribution $\overline{\tilde{u}_{i} \tilde{u}_{j}}$, the stochastic contribution $\overline{u_{i}^{\prime} u_{j}^{\prime}}$ and the total value for the baseline flow $\overline{u_{i} u_{j}}$. At the first downstream position, where the curved ramp starts $(x / H=0,4 d$ past the orifice), the injection is seen to result in a massive increase in total 'turbulence' activity, but much of this is clearly due to the periodic component, while the increase in the stochastic part is much lower. Moreover, the enhancement of the latter occurs, primarily, in the outer part of the boundary layer $y_{w} / H=0.2$, where the periodic component is also especially high, because of the strong cross-flow penetration of the jet, and where the coherent structures cause turbulencegenerating extra straining. This is the region in which the large phase-averaged structures depicted in figure 1 are located. Near the wall, $y_{w} / H=0.05$, there is a second peak, which is associated with the secondary motion induced by the primary outer vortices. As noted earlier, the rise in the stochastic part reflects extra turbulence production by unsteady straining. Close to the wall, the effect of the additional straining, relative to the background shear straining in the boundary layer, is very weak. In the outer region, however, the background shear straining is weak, while the periodic contribution is high, so that the effect of the latter on the stochastic turbulence is appreciable. At the next streamwise position $(x / H=0.95)$, the periodic contribution has already diminished dramatically, constituting only a modest part of the total. Remarkably, the total turbulence activity close to the wall is substantially reduced upon actuation. This is a direct consequence of the 
(a)

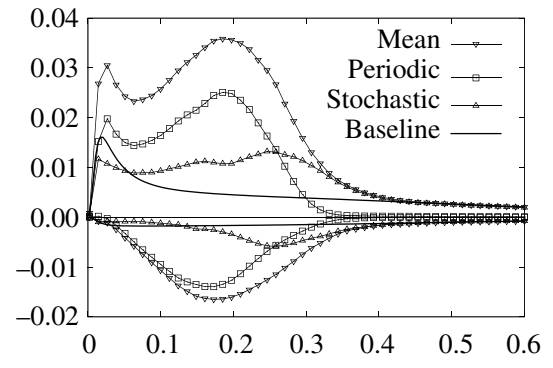

(c)

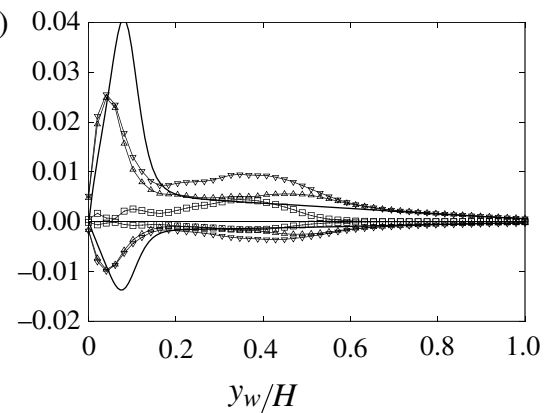

(b) 0.02

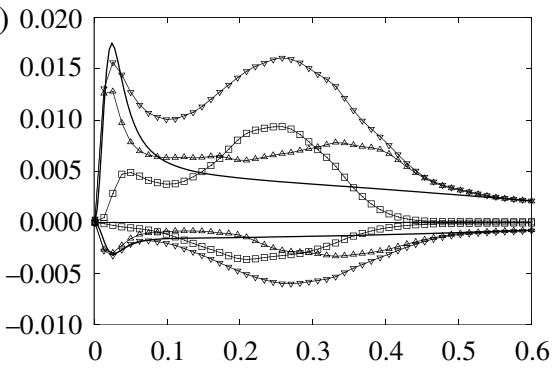

(d)

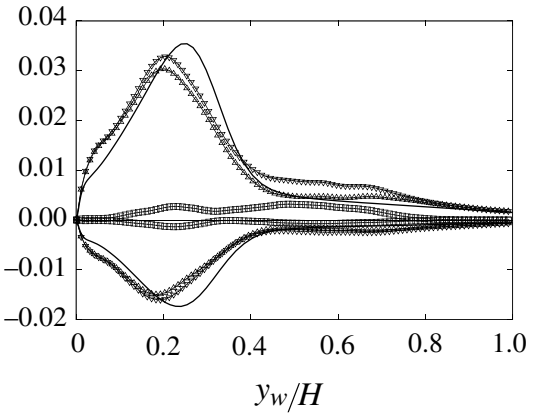

FIGURE 21. Reference actuated case $C_{\text {ref }}$, jet centre-plane. Profiles of total, coherent and stochastic stresses: mean $\overline{u_{i} u_{j}}$, periodic $\overline{\tilde{u}_{i} \tilde{u}_{j}}$, stochastic $\overline{u_{i}^{\prime} u_{j}^{\prime}}$, all normalised by $U_{i n}$. Positive values are for $i=1, j=1$ (streamwise stress); negative values are for $i=1, j=2$ (shear stress). (a) $x / H=0,(b) x / H=0.63,(c) x / H=1.25,(d) x / H=1.90$.

delay in the separation, and thus the delay in the rapid increase in turbulence associated with the boundary-layer separation and the $\mathrm{K}-\mathrm{H}$ vortices within the separated shear layer. At this position, the only direct (local) effect of the actuation is a weak accentuation of the stochastic turbulence in the outer region arising from the declining periodic straining. By $x / H=1.9$, the periodic component has diminished to an insignificant level, and the total stresses are virtually entirely due to the stochastic component, with differences relative to the baseline flow being weak.

In light of the above observations, it might be concluded that much of the potential of the actuation is wasted by an excessive penetration of the synthetic jet into the outer portion of the boundary, where any modifications to the flow are only weakly linked to the near-wall processes that govern separation. This suggests the wisdom of injection at a lower rate and at a shallower angle. Whether or not this assumption is correct will be investigated in the following sections.

The three-dimensional effects of the actuation process on the time-mean turbulence statistics are brought out in figure 22 by way of cross-flow plots of the same quantities shown in figure 21 for the streamwise stress at four streamwise locations close to the jet orifice. In these plots, the periodic part is shown on the left half of each plot, while the stochastic component is shown on the right. Several of the patterns are, essentially, similar in shape to those seen in the interaction of a boundary layer with a continuous cross-jet: a kidney-shaped set of contours that indicate the presence of two dominant streamwise vortices that draw boundary-layer fluid towards the centre-plane and outer fluid towards the wall. Immediately downstream of the orifice, most of the fluctuating motion is dominated by the periodic component, which reaches a maximum at the outer region of the vortex, $y_{w} / H=0.2$. The downward motion induced by this vortex around its side (around 

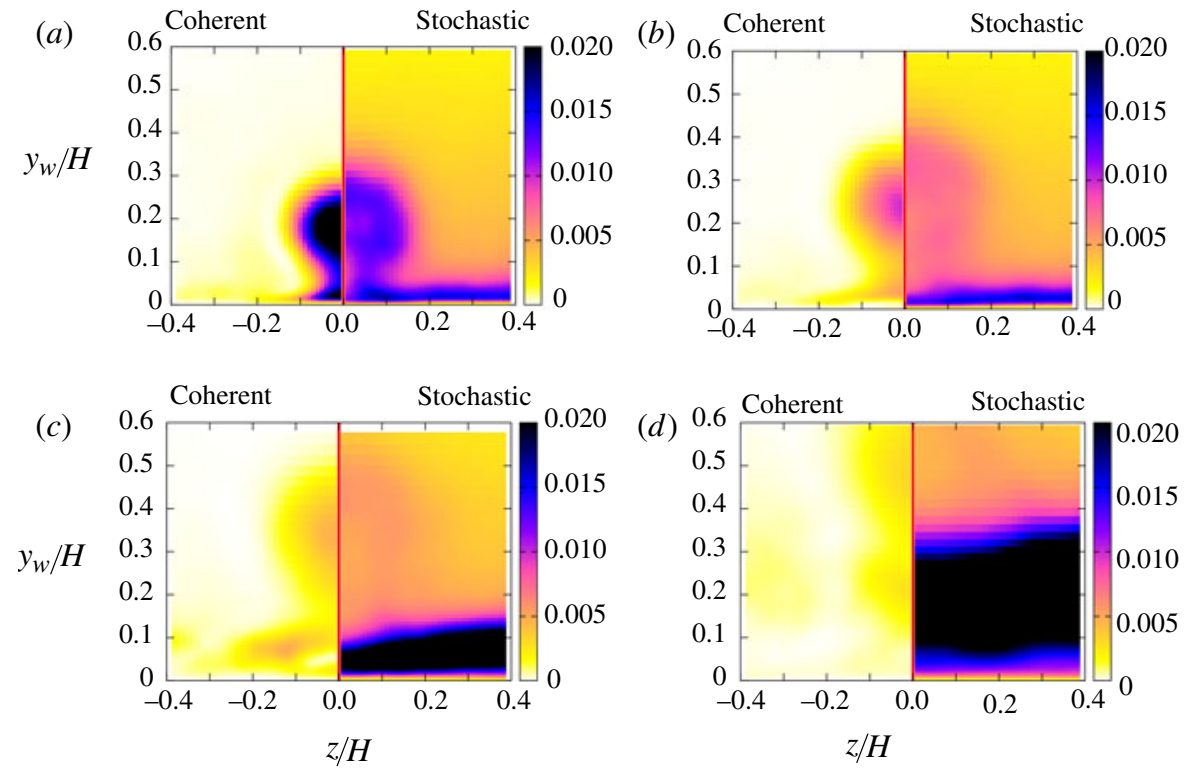

FIGURE 22. (Colour online available at journals.cambridge.org/flm) Reference actuated case $C_{r e f}$ : contours of time-average of periodic streamwise normal stress, $\overline{\tilde{u} \tilde{u}}$, and corresponding stochastic component $\overline{u^{\prime} u^{\prime}}$, normalised by $U_{i n}$, across four different streamwise positions. (a) $x / H=0,(b) x / H=0.63,(c) x / H=1.25,(d) x / H=1.90$.

$z / H=-0.2)$ transports high-momentum fluid towards the wall, while low-momentum fluid is convected upwards around the jet centre-plane. Further downstream the periodic component decays, especially close to the wall, and the stochastic component increasingly dominates. At the centre-plane, there is a distinct dip in the stochastic turbulence activity in the streamwise region, $x / H=0.63-1.25$, relative to that in the outer spanwise region, and this reflects the downstream shift of the separation around the centre-plane. These plots clearly convey the very limited spanwise extent of the region in which actuation is effective, $2 d$ at most. It is this fact that imposes the practical need for a much reduced inter-jet separation distance in efforts to achieve a higher level of control.

The present section has focused on one particular actuation scenario in detail, primarily because of the availability of experimental data for this case. The results and discussion have demonstrated the ability of the simulations to reproduce all essential physical features of the actuation process. It has illustrated the role of, and interaction between, the periodic and stochastic components, and led to the conclusion that the actuation parameters in question might not be optimal in terms of control authority of the separation process. In view of the above, the next two sections examine the benefit of altering the injection velocity and the injection angle, respectively.

\subsection{Effect of jet/boundary-layer-velocity ratio}

This section explores the consequences of reducing and increasing the maximum injection velocity, $V_{r}$, by $50 \%$ of the reference value. A supposition arising from the previous results is that increased injection velocity will not be advantageous, for the penetration at the reference value already appears to be excessive. More interesting is the question as to whether halving the injection velocity would result in the action being more effective in modifying the near-wall region at much lower expenditure of actuation energy. 

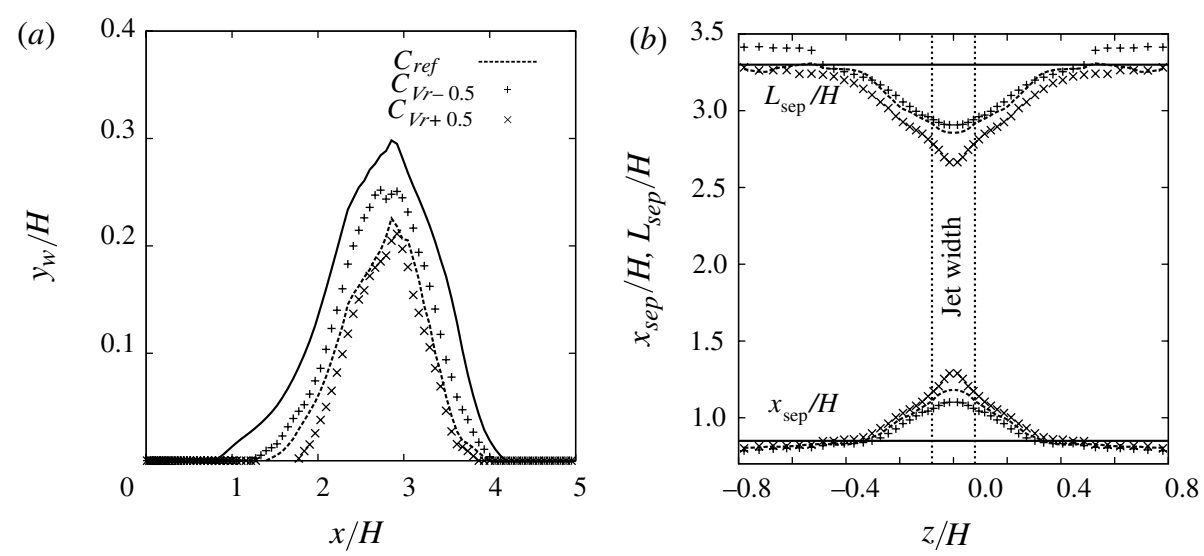

FIGURE 23. Sensitivity of separation zone to actuation strength. (a) Locus of zerostreamwise velocity in jet centre-plane; $(b)$ locus of separation location $x_{\text {sep }}$ and recirculation length $L_{r e f} ;-$, baseline case; -- -, $C_{r e f} ;+, C_{V r-0.5} ; \times, C_{V r+0.5}$.

A global view of the effects of the jet-velocity variation is first given in figure 23 . This shows the time-averaged separation and reattachment lines. The outcome is clear: an increase in injection velocity results in a more pronounced delay of separation and earlier reattachment, with the shift in reattachment extending over a larger spanwise distance. The effect is not dramatic but real. The results indicate, by implication, that the secondary perturbations near the wall, induced by the primary outer structures of the jet, are intensified by an increase of the injection velocity and thus translate to a slight increase in control authority.

Figure 19 shows that the momentum thickness on the centre-plane responds sensitively to the actuation strength. In particular, the blockage-induced elevation of $\theta$ is substantial at the highest actuation strength. As was argued in the previous section, this is not an advantageous feature, unless more than compensated by downstream mixing. While the delay of separation is evidently enhanced by increasing the actuation strength, this enhancement is confined to a rather narrow spanwise segment, and it can thus be argued that an increase of actuation intensity, over and above some minimum value, is not a very effective means of enhancing separation control.

The effect of $V_{r}$ on the time-averaged velocity and streamwise-normal and shear stresses is conveyed in figure 24 through profiles of the respective variables on the jet centre-plane at five streamwise locations for velocity and two for the Reynolds stresses. As the jet velocity is increased, there is a modest delay in the separation, with the near-wall gradient remaining positive over a slightly extended streamwise distance. However, a much more pronounced effect arises in the outer part of the boundary layer, $y_{w} / H=0.2-0.5$, especially at low $x / H$ values, wherein the velocity defect (trough) is intensified and also shifts outwards as the injection velocity increases, reflecting the more intense actuation and higher penetration. The effect on the downstream velocity profiles is minor, consistent with the earlier observation that the principal effect of the actuation is confined to the region upstream and immediately downstream of the separation line. As regards the Reynolds stresses, an increase in $V_{r}$ results in a corresponding increase in total turbulence activity in the outer parts of the boundary layer - clearly conveyed in the plot for $x / H=1.25$. There is also a noticeable effect on the turbulence activity close to the wall: while the peak decreases with increasing $V_{r}$, the more important effect is the elevation of turbulence in 


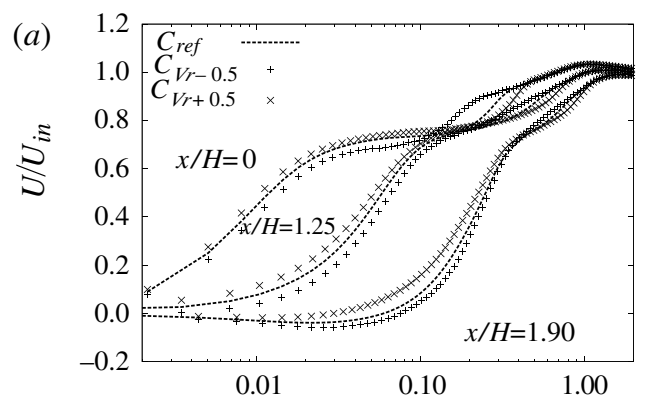

(b)
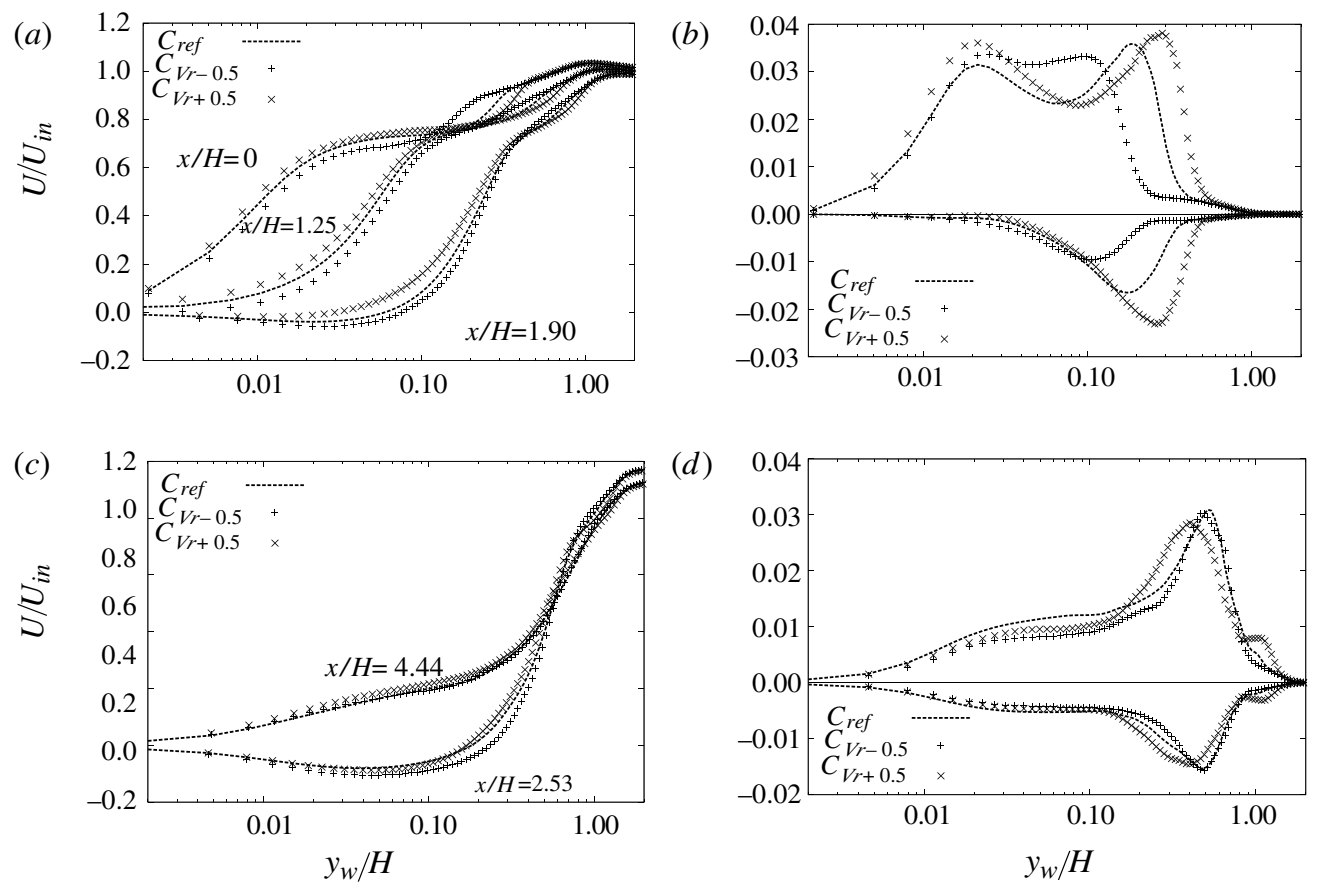

FIGURE 24. Sensitivity of flow properties to actuation strength in jet centre-plane. $(a, c)$ Streamwise velocity, $U / U_{i n} ;(b)$ streamwise normal stress $\overline{u u} / U_{i n}^{2}$ and shear stress $\overline{u v} / U_{i n}^{2}$ for $x / H=1.25 ;(d)$ the same as $(b)$ but at $x / H=2.53$.

the region $x / H<0.05$, because it is this elevation, albeit slight, that is most likely to be responsible for the delay in separation as $V_{r}$ increases. The origin of this elevation will be clarified below. As is the case for the velocity, once separation is well established, the influence of $V_{r}$ on the turbulence level is minor, although there is clear evidence of the height of the separated shear layer reducing, as reflected by the shear-induced turbulence peaks being closer to the wall. This presumably reflects primarily the delayed separation, which then results in a thinner reverse-flow region.

The effect of injection velocity on the cross-flow properties, especially the lateral region of influence, is conveyed in figure 25, which corresponds to figure 22 for the reference case. First, the lower injection velocity results, as expected, in the perturbed region being much closer to the wall, reflecting lower penetration. Second, the lower injection velocity is also seen to generate, in most areas, lower fluctuations, both periodic and stochastic, away from the wall. Close to the wall, however, the periodic contribution is higher, because the large periodic structures of the jet are also much closer to the wall. Third, the lateral extent of the perturbation is substantially larger with increasing injection velocity, and this results in correspondingly larger lateral effects on the separation and especially reattachment lines (figure 23). Fourth, at the highest injection velocity, the outer and inner parts are almost detached from each other, while there is a much greater level of wall-normal coherence or continuity in properties at the lowest velocity. Finally, it is observed that the higher injection velocity results in a more intensive streamwise vortex. This has two attendant consequences: first the perturbations and secondary vortices induced close to the wall are more vigorous, leading to higher fluctuation levels; second, the vertical motion transports highly turbulent fluid from the primary vortex downwards, towards the wall, leading to a 

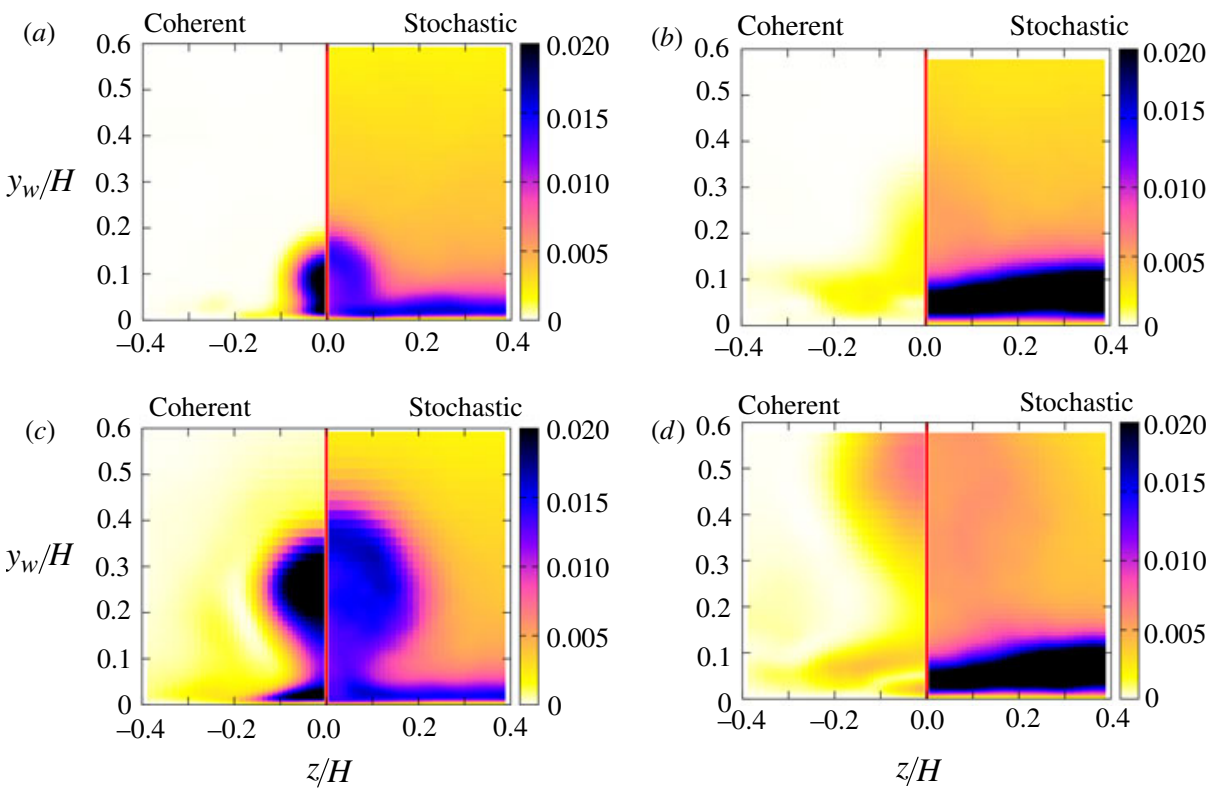

FIGURE 25. (Colour online) Sensitivity of cross-sectional fields of streamwise stress to actuation strength (corresponding to figure 22). (a,b) $C_{V r-0.5} ;(c, d) C_{V r+0.5} ;(a, c) x / H=0$; $(b, d) x / H=1.25$.

further elevation of near-wall turbulence. However, the result of both processes is rather local, being confined to a narrow region around the jet centre-plane. Hence, the overall effect on the separation process is only modest.

\subsection{Effect of injection angle}

Intuitively, one might assume that a flow-oriented injection, at an acute angle, would be beneficial in two respects: first, such injection imparts additional streamwise momentum to the flow, and it does so preferentially in the near-wall region; second, penetration is lower, and the unsteady large-scale structures are closer to the wall. On the other hand, one might also expect somewhat weaker secondary motions, found to be advantageous in the above investigation of the effects of the jet velocity. In a similar vein, injection against the flow might be expected to generate higher levels of turbulence close to the injection and a larger spanwise penetration of actuation and hence a wider region across which separation is affected - due to a blooming-jet-like process. Earlier experience with variations in the injection angle (e.g. Garcillan et al. 2006) suggest that injection at a shallow angle, in the direction of the flow, is the most effective, in so far as it generates strong and longlived streamwise vortices. The physical scenario proposed is that the realignment of the jet is modest, thus securing a high level of coherence of the vortical secondary motion over a longer streamwise distance. It is against this background that the present section investigates the effects of injection angle through simulations of injection at 45 and 135 degrees relative to the streamwise direction ( $C_{\alpha-45}$ and $C_{\alpha+45}$, respectively).

The global effect of the injection angle on the separation and reattachment is shown in figure 26 . Blowing upstream $\left(135^{\circ}\right)$ is seen to be substantially more effective at reducing the separation bubble than blowing downstream $\left(45^{\circ}\right)$, with a maximum reduction of $35 \%$ in the length of the recirculation region at the centre-plane. Moreover, counter-flow injection results in a materially larger lateral spread of the separation delay, with the effect 

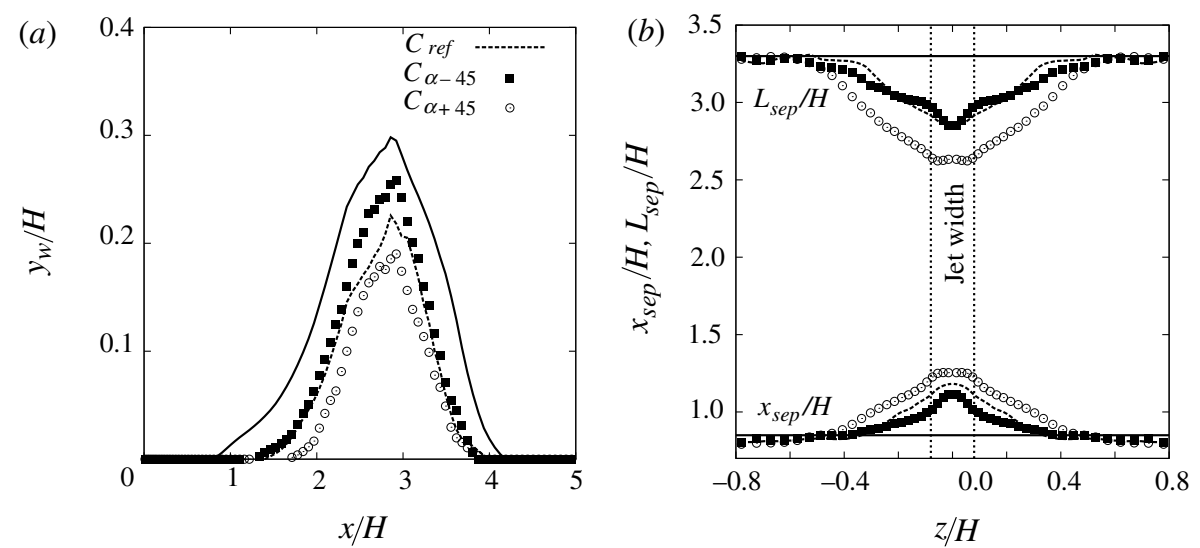

FIGURE 26. Sensitivity of separation zone to actuation angle. (a) Height of zero-streamwise velocity locus above the lower wall in jet centre-plane; $(b)$ locus of separation location $x_{s e p}$ and recirculation length $L_{s e p} ;-$, baseline case; ---, $C_{r e f} ; \circ, C_{\alpha+45} ; \mathbf{\square}, C_{\alpha-45}$.

extending up to $z / H= \pm 0.5$, or $z / d= \pm 3$. This is consistent with the blooming-jet notion alluded to earlier and observed with dramatic effects in preliminary simulations by the present authors for actuation into a laminar boundary layer. In contrast, and as is also expected, co-flow injection leads to a narrowing of the region in which separation is delayed, reflecting a greater coherence of the jet region. The effect of the injection on the reattachment is not significant, however, although there is a slight upstream shift at both $45^{\circ}$ and $135^{\circ}$.

Figure 19 shows that the actuation angle has a dramatic effect on the momentum thickness downstream of the actuation. Co-flow actuation actually results in a slight reduction in the momentum thickness, while counter-flow actuation leads to a substantial increase. In view of earlier comments, it might be expected that co-flowing actuation would yield the most effective control authority. As shown above, this is not the case, and the reason is, evidently, the strong mixing, especially in the spanwise direction, that counter-flow injection provokes.

While all three cases lead, globally, to similar reductions in separation, there are some major differences in the detailed mechanisms involved. This emerges from figure 27, which gives profiles of the streamwise velocity at five streamwise positions and two Reynolds-stress components at two streamwise positions in the jet centre-plane.

Counter-flow injection is seen to result in much higher levels of turbulence energy at $x / H=1.25$, both in the inner and the outer parts of the boundary layer, in contrast to the other two cases, and hence in the most pronounced delay in separation. This mode of injection also results in the largest obstruction (wake) in the wall-normal layer around $y_{w} / H=0.1$, simply because of the injection of counter-flow momentum. Curiously, at first sight, the shear stress for this case is relatively low around $y_{w} / H=0.05-0.1$, but reference to the velocity profiles reveals that this is within the wake region of the jet, an area in which the shear strain is relatively low. The high level of $\overline{u u}$ is due mainly to the coherent motion, but the shear stress is much less affected than the coherent component and dominated by the stochastic processes. In co-flow actuation, the outer turbulence peak is absent, as penetration is much less pronounced, with the jet hugging the wall. The level of fluctuations is similar to the normal injection case. Here, the shear stress at $y_{w} / H=0.1$ is highest, and this goes hand-in-hand with the weaker velocity wake and higher shear 


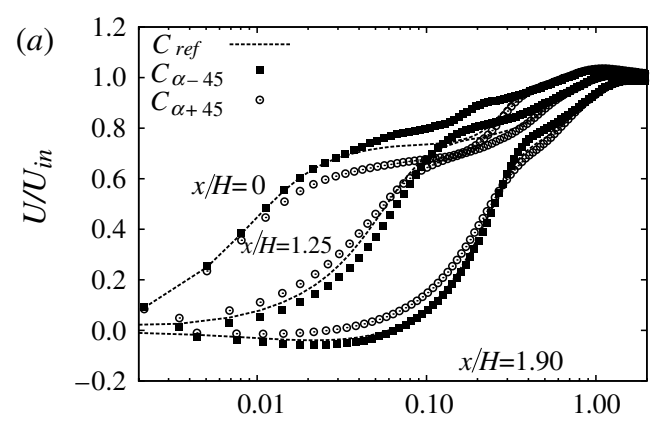

(b)
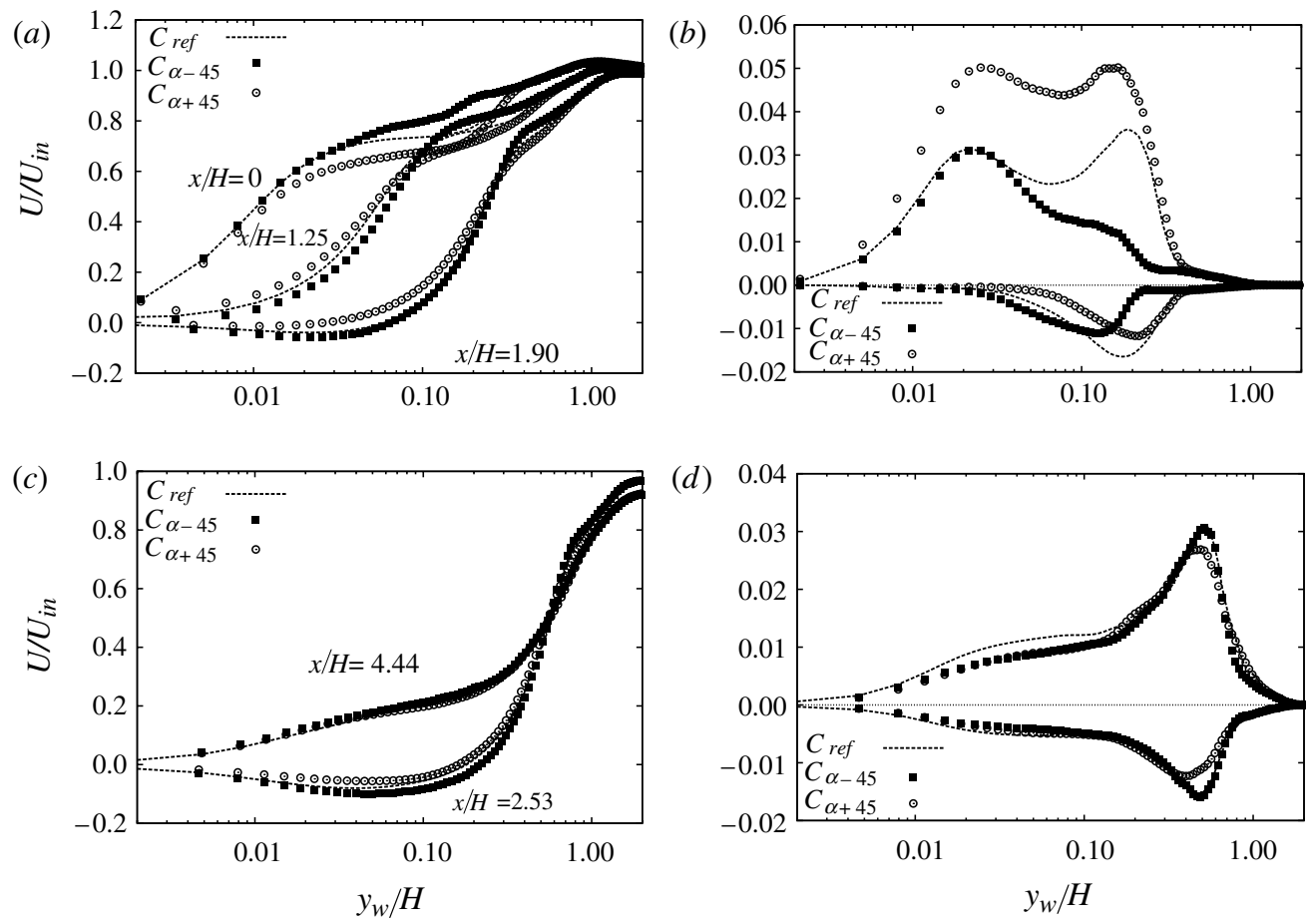

FIGURE 27. Sensitivity of time-mean flow properties to actuation angle in jet centre-plane. $(a, c)$ Streamwise velocity $U / U_{i n} ;(b)$ streamwise normal stress $\overline{u u} / U_{\text {in }}^{2}$ and shear stress $\overline{u v} / U_{\text {in }}^{2}$ at $x / H=1.25 ;(d)$ the same as $(b)$ but at $x / H=2.53$.

strain in this region. However, the overall effect on the near-wall layer is not enhanced relative to wall-normal injection, and the fact that co-flow injection is least effective in terms of spanwise control makes this mode of injection least attractive.

The superior effectiveness of the counter-flow injection is confirmed in figure 28 . Counter-flow injection results in high levels of turbulence activity over a large wallnormal as well as spanwise area. The high-turbulence tongue extending to the wall at around $z / H=0.1-0.3$ (right half of plot of figure $28 c$ ) is especially noteworthy, and the broadening of the separation line in figure 26 is clearly associated with it. In co-flowing injection, the effects of the actuation is clearly much more confined, laterally as well as in wall-normal direction. While this injection mode generates a tight, high-intensity streamwise vortex, with high spanwise velocity towards the centre-plane near the wall, the turbulence level at the wall is low, relative to that in counter-flow injection, and thus the separation control is less effective.

The major differences between the co-flow and counter-flow actuations are clearly revealed upon juxtaposing figures 29 and 30, and contrasting both with figure 16 for the wall-normal actuation, all showing the phase-varying periodic fluctuations in streamwise and wall-normal velocity. In co-flow injection (figure 29), the periodic fluctuations are weaker and the regions of intense fluctuations smaller than in wall-normal actuation. In particular, positive near-wall fluctuations are narrow and confined to a smaller portion of the actuation cycle $(\phi=1.5-2 \pi)$. This is linked directly to the weaker transverse circulation induced by the actuation and hence lower convective transport of high- 

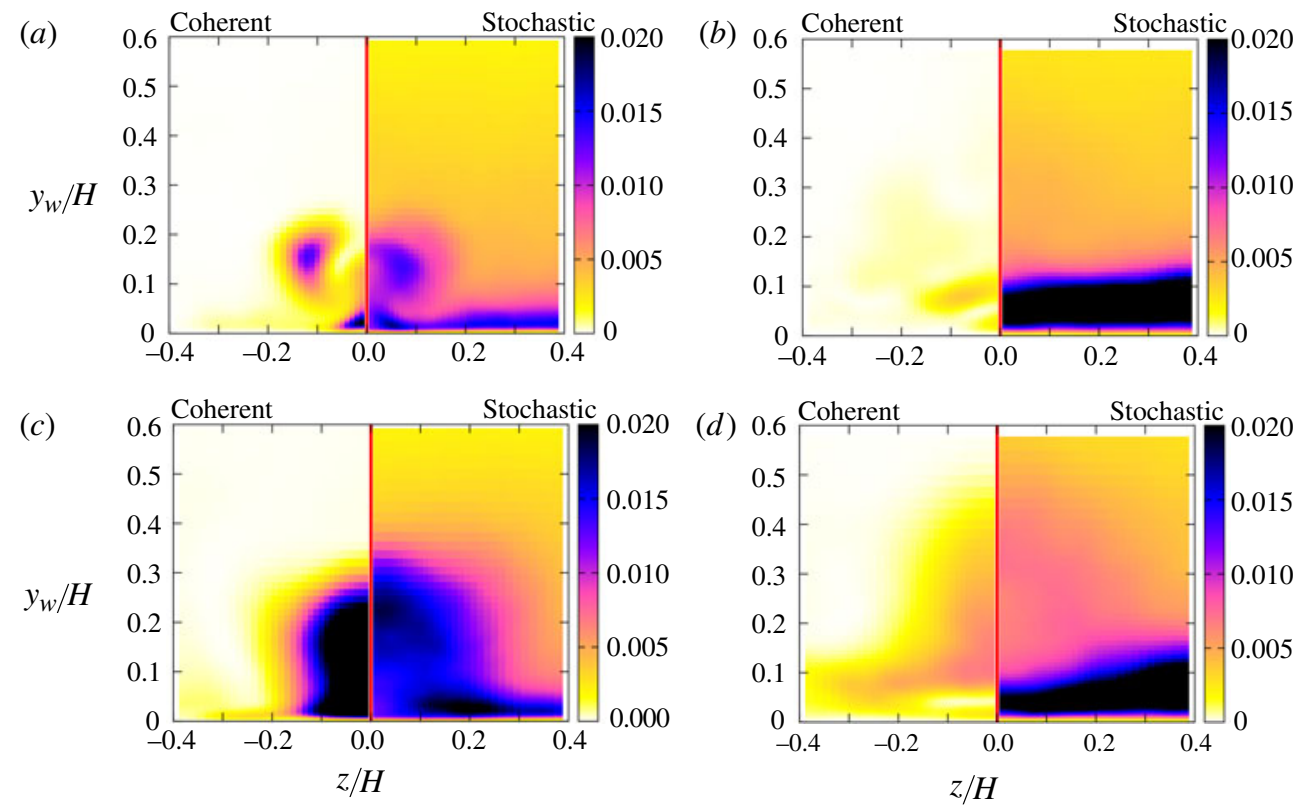

FIGURE 28. (Colour online) Sensitivity of cross-flow fields of the streamwise normal stress to the actuation angle (corresponding to figure 22). (a,b) $C_{\alpha-45} ;(c, d) C_{\alpha+45} ;(a, c) x / H=0$; $(b, d) x / H=1.25$.

momentum fluid from outer regions towards the wall. In the remainder of the cycle, the near-wall region is dominated by negative streamwise fluctuations which are ineffective in terms of separation delay. During the ejection phase, for the counter-flow actuation (figure 30), streamwise momentum is injected into the outer wall-normal region, and this is clearly reflected by regions of positive streamwise fluctuation away from the wall at $\phi=4 \pi / 8-6 \pi / 8$, in contrast to the corresponding conditions in wall-normal injection (cf. figure 16). In counter-flow injection (figure 30 ), the periodic motions are clearly much more vigorous and extend far wider in the spanwise direction. The blooming-jet behaviour arising during the ejection phase is especially well shown by the wall-normal velocity at $\phi=4 \pi / 8$. The actuation generates pronounced downward motion, both during the ejection and suction phases, and this enhances the formation of extensive regions of positive fluctuations over a substantial proportion of the actuation cycle, which persist over a significantly larger downstream distance than the other actuation scenarios. These images are thus fully consistent with the enhanced separation-control characteristics indicated by the statistical data.

Overall, the actuation tends to elevate $\theta / H$ (figure 19), especially immediately downstream of the jet, with one notable exception - namely forward injection at $45^{\circ}$. This elevation reflects primarily the major thickening of the flow by the blocking influence of the jet and the wake immediately behind it. Once this thickening has occurred, it tends to decrease only modestly as the flow evolves on the flat portion preceding the ramp, subject to modest acceleration. As shown already, the jet induces substantial streamwise vortices, which tend, through cross-flow convection and associated turbulence, to promote cross-flow mixing and thus elevate the near-wall velocity. It is this elevation, associated with turbulence (both coherent and stochastic) that promotes increased resistance to separation. In fact, it can be argued that the optimum combination is low jet-induced 

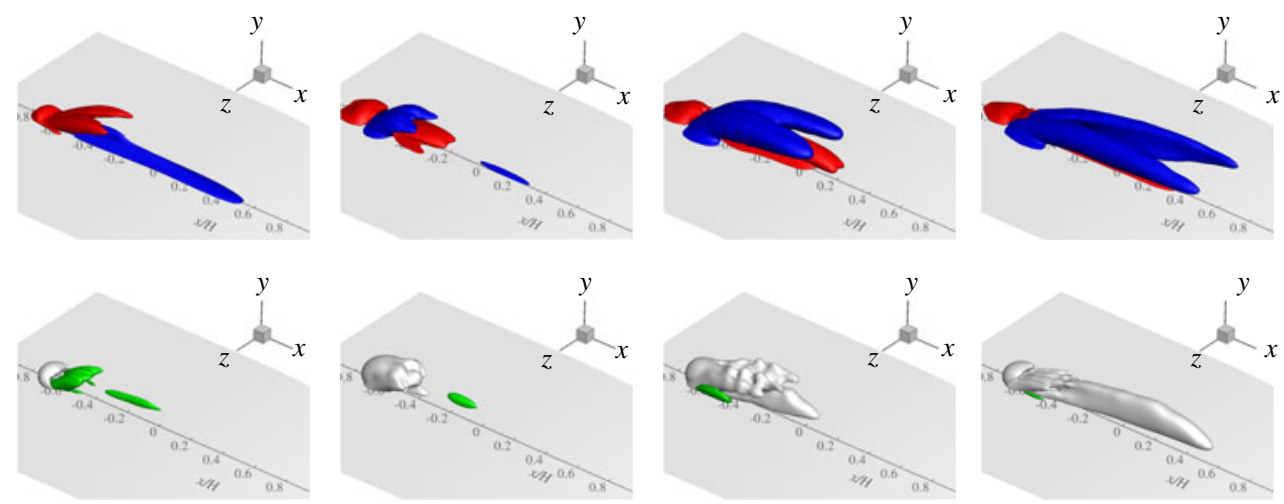

0

$2 \pi / 8$

$4 \pi / 8$

$6 \pi / 8$
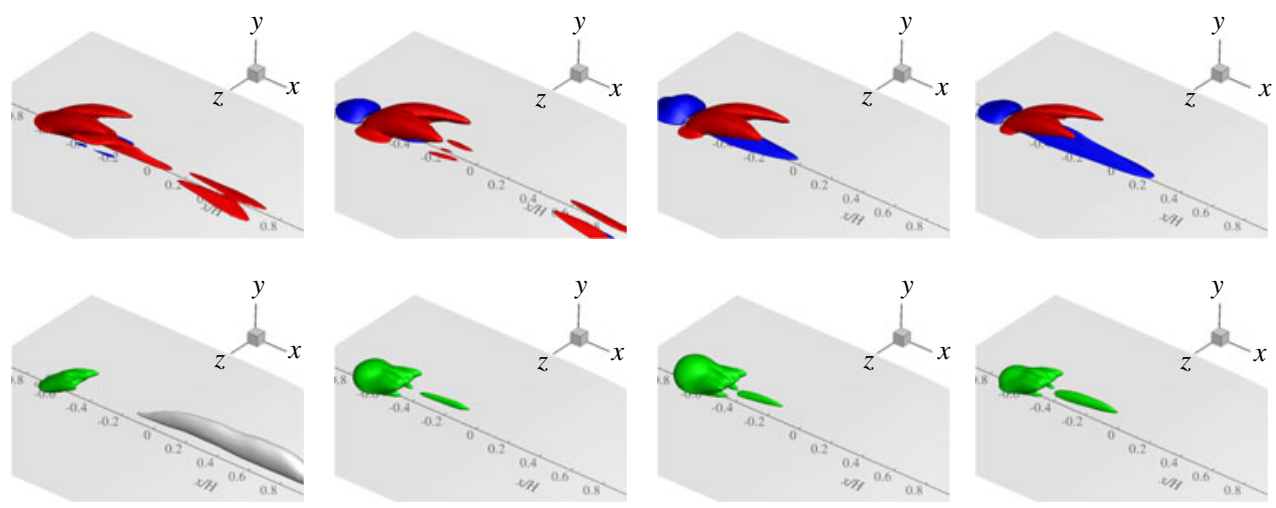

$10 \pi / 8$

$12 \pi / 8$

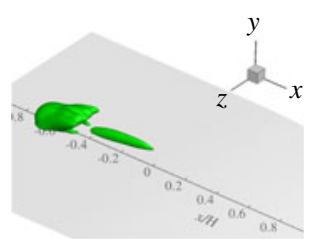

$14 \pi / 8$

FIgURE 29. Actuated case $C_{\alpha-45}$ : sensitivity of streamwise periodic motion to injection angle: isosurfaces of $\tilde{u}= \pm 0.1 U_{\text {in }}(+0.1$ in blue and -0.1 in red $)$, and of $\tilde{v}= \pm 0.1 U_{\text {in }}(+0.1$ in grey and -0.1 in green), at 8 equally spaced phase values (progressing left to right and top to bottom) covering a whole cycle.

momentum thickening coupled with intensive downstream mixing. As might be expected, momentum thickening is most pronounced at high injection velocity and counter-flow injection. In both cases, the obstruction to the oncoming flow is large, and thus thickening is maximised. In contrast, injection at $45^{\circ}$ hardly affects $\theta$ and might be regarded as the most advantageous. However, this fails to take into account the lateral spread of the jet and thus its lateral region of influence on the separation, as explained in an earlier part of the discussion.

\section{Conclusions}

The study has investigated the fluid-mechanic characteristics of a turbulent boundary layer separating from a rounded ramp and the sensitivity of the separation process to periodic perturbations created by the injection of synthetic round jets. Conclusions emerging from the study are most appropriately divided into two groups, respectively focusing on global and local characteristics, the former oriented towards the technological exploitation of synthetic-jet actuation, and the latter towards fundamental properties. 


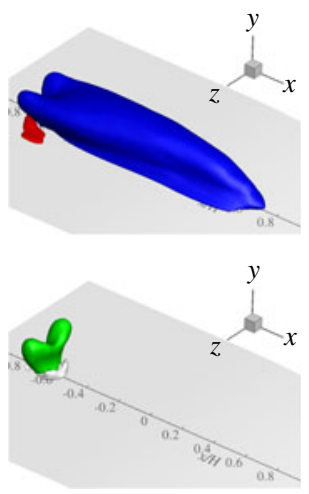

0
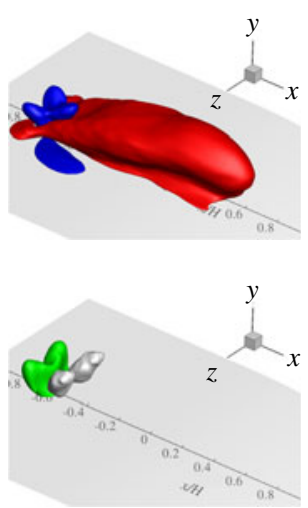

$8 \pi / 8$
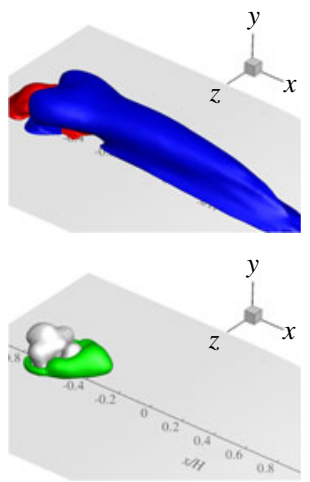

$2 \pi / 8$
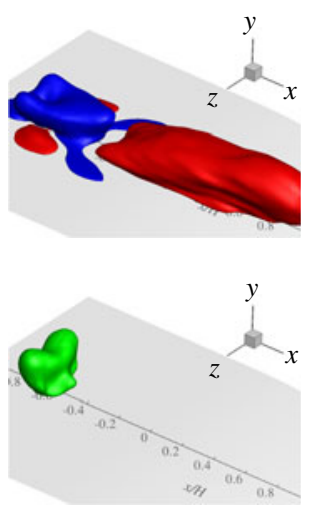

$10 \pi / 8$
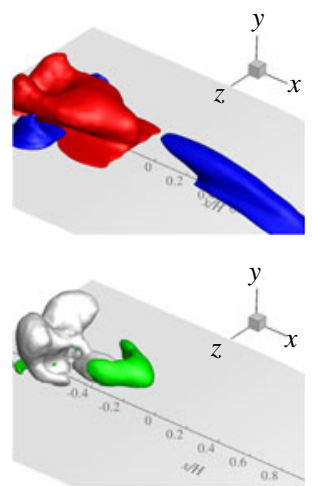

$4 \pi / 8$
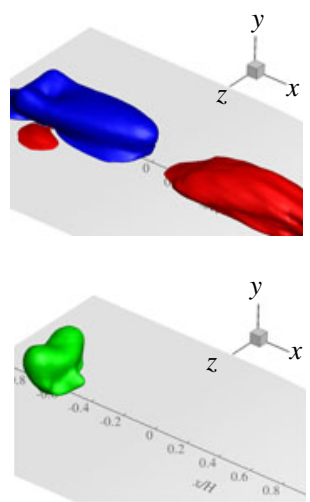

$12 \pi / 8$
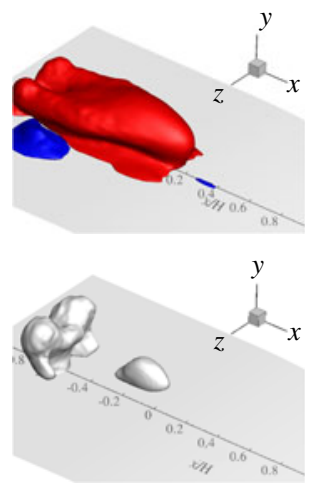

$6 \pi / 8$
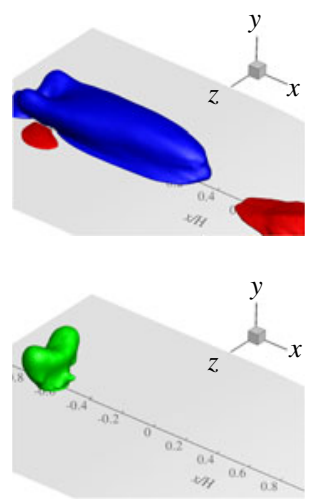

$14 \pi / 8$

FIGURE 30. As figure 29 but $C_{\alpha+45}$.

Of greatest practical interest is the effect of the actuation on the size of the separated region, effectively characterising the control authority of the actuation. The study has shown that the present geometric arrangement is not conducive to a strong control authority, because the jet orifices are located 7 diameters upstream of the nominal separation location and are laterally separated by 10 diameters, both reflecting experimental constraints. Nevertheless, there is an appreciable reduction of around $10-20 \%$ in the length of the recirculation zone, depending on the actuation mode and up to $40 \%$ reduction in the thickness of the reverse-flow layer. Despite the actuationinduced increase in momentum thickness, associated with blockage and adverse pressure gradient in the near-wake of the jets, the substantially enhanced level of mixing behind the actuated jet results in a net benefit of separation delay and also in a modest backward shift in the mean reattachment location. The most effective actuation involves counter-flow injection, here at $135^{\circ}$ relative to the boundary-layer direction. High injection velocity is also beneficial, but to a lesser extent.

The above figures refer to a region around the jet centre-plane, while the lateral effects are more modest, with the counter-flow injection yielding the best result. Depending on the actuation mode, the lateral region of influence on the separation extends to around 2-3 orifice diameters on either side of the centre-plane, suggesting the need for orifices to be separated by around 2-5 diameters, rather than 10 as done here, to achieve a credible spanwise uniformity of the control. This is contingent on the orifices remaining 
in the present streamwise location. However, there are clear indications, derived from an examination of the periodic and stochastic contributions to the statistics, that the jets should be placed closer to the separation location, but then the spanwise separation must also be reduced.

Although the present paper does not deal explicitly with the sensitivity of the control to the actuation frequency - the one considered, corresponds to the accepted shedding mode, $S t_{H}=0.2-$ it is worth noting here that unreported simulations do not provide evidence of a significant sensitivity in this respect. This is in contrast to slot jets, in which the spanwise uniformity of the actuation favours a pronounced resonance with the two-dimensional stability modes of the separated shear layer. Spectra included in the paper for the baseline case indicate that the most energetic motions indeed correspond to $S t_{H}=0.2$, relative to $S t_{H}=1-2$ in the attached boundary layer. Thus, if there is any sensitivity to the actuation frequency, it is likely that the choice $S t_{H}=0.2$ is close to optimal.

In terms of fluid-mechanic fundamentals, the study has highlighted, first, the complex nature of the separation processes in the unperturbed flow. Thus, the time-averaged recirculation zone is very thin at its upstream end and grows gradually. Moreover, the separated shear layer is characterised by very high levels of turbulence activity, reflecting the unsteady separation behaviour, which is linked to temporal scale in the boundary layer, as well as the reverse propagation of disturbances linked to the $\mathrm{K}-\mathrm{H}$ instability in the separated shear layer. The turbulence-energy budgets indicate a state in the shear layer that is very far from turbulence equilibrium, with production being 2-3 times higher than dissipation.

In the presence of actuation, an influential process by which the actuation exercises separation control is by causing additional mixing due to streamwise vortices and additional stochastic turbulence provoked by unsteady straining. The time-averaged results present a picture that is akin to that pertaining to continuous-jet injection: thus, the dominant process is the creation of a vigorous pair of vortices in the outer part of the boundary layer that causes wall-normal exchange of momentum. This is aided by much weaker vortices close to the wall, induced by the outer vortices. The effect is most pronounced, especially laterally, in the case of counter-flow injection. Second, an important observation relates to the relative contributions of actuation-induced periodic and stochastic fluctuations to the turbulent stresses. In the region within around 5-7 orifice diameters downstream of the injection - that is, upstream of separation - there is a strong dominance of the contribution of the periodic component to both the streamwise stress and the shear stress, both being elevated up to 4-to-5-fold. This is accompanied by a more modest elevation of the stochastic component. The above elevation occurs primarily in the outer part of the boundary layer into which the ejected unsteady vortical structure penetrates due to the high speed of the ejected fluid. The enhancement is much more modest closer to the wall, where secondary vortices are induced. As the actuated boundary layer reaches the nominal separation line, the turbulence levels close to the wall are relatively low, as the boundary layer is firmly attached and more stable than the separating layer in the absence of actuation. In the outer layer, both the periodic and stochastic extra contributions are still noticeable, but much reduced relative to those further upstream. This strengthens the conclusion that the position of the orifices has to be closer to the separation location, perhaps as close as 1-2 diameters upstream of the separation, to derive more benefits from the actuation. Once separation has occurred, the influence of the actuation on the turbulence field is minor - that is, most of the control is derived from a delay of the separation.

Phase-averaged fields of the periodic streamwise- and wall-normal velocity perturbations have brought to light some important differences among the actuation 
scenarios. An important distinction relates to the predominance of positive near-wall periodic streamwise perturbations in some scenarios, which then translate to the largest phase- and time-averaged forward shifts in separation location. This is seen especially at high actuation intensity and counter-flow actuation. This process adds to the benefits of enhanced mixing, especially in these two actuation cases.

The authors acknowledge the financial support granted to them by Airbus (UK) and the UK Engineering and Physical Sciences Research Council (EPSRC). Some of the simulations reported herein were obtained with resources from the UK Turbulence Consortium, supported by EPSRC. Finally, the authors are grateful to Dr S. Zhong and S. Zhang, of the University of Manchester, for providing their experimental data in digital form.

\section{REFERENCES}

ARAM, E., MitTAL, R. \& CATtAFEStA, L. 2010 Simple representations of zero-net mass-flux jets in grazing flow for flow-control simulations. Intl J. Flow Control 2 (2), 109-125.

AVDis, A., LARDEAU, S. \& LESCHZINER, M. 2009 Large eddy simulation of separated flow over a two-dimensional hump with and without control by means of a synthetic slot-jet. Flow Turbul. Combust. $\mathbf{8 3}$ (3), 343-370.

CAter, J. E. \& Soria, J. 2002 The evolution of round zero-net-mass-flux jets. J. Fluid Mech. 472, 167-200.

DANDOIS, J., GARNIER, E. \& SAGAUT, P. 2006 Unsteady simulation of a synthetic jet in a crossflow. AIAA J. 44 (2), 225.

DAndois, J., GARnier, E. \& SAGAUT, P. 2007 Numerical simulation of active separation control by a synthetic jet. J. Fluid Mech. 574, 25-58.

DeJOAn, A. \& LESCHZINER, M. A. 2004 Large eddy simulation of periodically perturbed separated flow over a backward-facing step. Intl J. Heat Fluid Flow 25 (4), 581-592.

Fishrool, G. M. \& LESCHZINER, M. A. 2009 Stability bounds for explicit fractional-step schemes for the Navier-Stokes equations at high Reynolds number. Comput. Fluids 38 (6), 1289-1298.

Fröhlich, J., Mellen, C., Rodi, W., Temmerman, L. \& Leschziner, M. A. 2005 Highly resolved large-eddy simulation of separated flow in a channel with streamwise periodic constrictions. J. Fluid Mech. 526 (-1), $19-66$.

Garcia-Villalba, M., Li, N., Rodi, W. \& Leschziner, M. A. 2009 Large-eddy simulation of separated flow over a three-dimensional axisymmetric hill. J. Fluid Mech. 627, 55-96.

Garcillan, L., Liddle, S., Sunneechurra, K., Crowther, B., Zhong, S. \& Wood, N. 2006 PIV measurements of the effect of pitch and skew on a circular orifice synthetic jet in a turbulent boundary layer. AIAA Paper 318, 2006.

Germano, M., Piomelli, U., Moin, P. \& CABot, W. H. 1991 A dynamic subgrid-scale eddy viscosity model. Phys. Fluids A 3, 1760.

Gharib, M., RAmbod, E. \& ShAriff, K. 1998 A universal time scale for vortex ring formation. J. Fluid Mech. 360, 121-140.

GLEZER, A. 1981 An experimental study of a turbulent vortex ring. PhD thesis, California Institute of Technology.

Glezer, A. \& Amitay, M. 2002 Synthetic jets. Annu. Rev. Fluid Mech. 34 (1), 503-529.

Gordon, M. \& SORIA, J. 2002 PIV measurements of a zero-net-mass-flux jet in cross flow. Exp. Fluids 33 (6), $863-872$.

Greenblatt, D., Paschal, K. B., Yao, C., Harris, J., Schaeffler, N. W. \& Washburn, A. E. 2006 Experimental investigation of separation control. Part 1. Baseline and steady suction. AIAA J. 44 (12), 2855.

HASAN, M. A. Z. \& KHAN, A. S. 1992 On the instability characteristics of a reattaching shear layer with nonlaminar separation. Intl J. Heat Fluid Flow 13 (3), 224-231.

Huss AIn, A. 1983 Coherent structures: reality and myth. Phys. Fluids 26, 2816.

INAGAKI, M., KONDOH, T. \& NAGANO, Y. 2005 A mixed-time-scale SGS model with fixed model-parameters for practical LES. Trans. ASME: J. Fluids Engng 127, 1. 
JABBAL, M. \& ZhONG, S. 2008 The near wall effect of synthetic jets in a boundary layer. Intl J. Heat Fluid Flow 29 (1), 119-130.

JimÉnez, J., Hoyas, S., Simens, M. P., Mizuno, \& Y., 2010 Turbulent boundary layers and channels at moderate Reynolds numbers. J. Fluid Mech. 1-26.

Krishnan, V., SQUiRes, K. D. \& Forsythe, J. R. 2006 Prediction of separated flow characteristics over a hump. AIAA J. 44 (2), 252.

Leschziner, M. A. \& LARdeAU, S. 2011 Simulation of slot and round synthetic jets in the context of boundary-layer separation control. Philos. Trans. Royal Soc. A: Math. Phys. Engng Sci. 369, 1495-1512.

LiLLY, D. K. 1992 A proposed modification of the Germano subgrid-scale closure method. Phys. Fluids A 4, 633.

LUND, T. S., WU, X. \& SQUIRES, K. D. 1998 Generation of turbulent inflow data for spatially-developing boundary layer simulations. J. Comput. Phys. 140 (2), 233-258.

Mittal, R. \& Rampunggoon, P. 2002 On the virtual aeroshaping effect of synthetic jets. Phys. Fluids 14, 1533.

NeUmann, J. \& Wengle, H. 2004 Coherent structures in controlled separated flow over sharp-edged and rounded steps. J. Turbul. 5 (22), 1-24.

OpPenheIM, A. V. \& SCHAFER, R. W. 2007 Discrete-time signal processing. Pearson Education; 3 edition (31 Dec 2007), ISBN-13:978-0132067096.

OzAwA, T., Lesbros, S. \& Hong, G. 2010 LES of synthetic jets in boundary layer with laminar separation caused by adverse pressure gradient. Comput. Fluids 39, 845-858.

Pamart, P. Y., Dandois, J., Garnier, E. \& SAgaut, P. 2010 Large Eddy Simulation Study of Synthetic Jet Frequency and Amplitude Effects on a Rounded Step Separated Flow, 5th AIAA Shear Flow Control Conference, Chicago, Illinois, 28/06 to 01/07 2010.

Raju, R., ARAM, E., MitTal, R. \& CatTafesta, L. 2009 Simple models of zero-net mass-flux jets for flow control simulations. Intl J. Flow Control 1 (3), 179-197.

Ravi, B. R., MitTAL, R. \& NAJJAR, F. M. 2004 Study of three-dimensional synthetic jet flow fields using direct numerical simulation. AIAA Paper 51, 61801.

RHIE, C. M. \& CHOW, W. L. 1983 Numerical study of the turbulent flow past an aerofoil with trailing edge separation. AIAA J. 21 (11), 1525-1532.

Rizzetta, D. P., Visbal, M. R. \& StaneK, M. J. 1999 Numerical investigation of synthetic-jet flow fields. AIAA $J .37$ (8).

Rumsey, C. L., Gatski, T. B., Sellers, W. L., Vatsa, V. N. \& Viken, S. A. 2006 Summary of the 2004 computational fluid dynamics validation workshop on synthetic jets. AIAA J. 44 (2), 194.

SAFFMAN, P. G. 1981 Dynamics of vorticity. J. Fluid Mech. 106, 49-58.

SAU, R. \& MAHESH, K. 2008 Dynamics and mixing of vortex rings in crossflow. J. Fluid Mech. 604, 389-409.

SCHAEFFLER, N. W. \& JENKINS, L. N. 2006 Isolated synthetic jet in crossflow: experimental protocols for a validation dataset. AIAA J. 44 (12), 2835.

Smith, B. L. \& Glezer, A. 1998 The formation and evolution of synthetic jets. Phys. Fluids 10, 2281.

Smith, B. L. \& SwIFT, G. W. 2003 A comparison between synthetic jets and continuous jets. Exp. Fluids 34 (4), 467-472.

SONG, S. \& EATON, J. K. 2004 Reynolds number effects on a turbulent boundary layer with separation, reattachment, and recovery. Exp. Fluids 36 (2), 246-258.

UdAykumar, H. S., Mittal, R., Rampunggoon, P. \& Khanna, A. 2001 A sharp interface Cartesian grid method for simulating flows with complex moving boundaries. J. Comput. Phys. 174 (1), 345-380.

WU, D. K. L. \& LESCHZINER, M. A. 2009 Large-eddy simulations of circular synthetic jets in quiescent surroundings and in turbulent cross-flow. Intl J. Heat Fluid Flow 30 (3), 421-434.

You, D., HAM, F. \& MoIN, P. 2008 Discrete conservation principles in large-eddy simulation with application to separation control over an airfoil. Phys. Fluids 20, 101515.

Zaman, K. \& Milanovic, I. 2002 Synthetic jets in cross-flow. Part II. Jets from orifices of different geometry. In 33rd AIAA Fluid Dynamics Conference and Exhibit AIAA Paper 2002-3715, Orlando, FL, 23-26 June.

ZHANG, S. \& ZHONG, S. 2010 An experimental investigation of turbulent flow separation control by an array of synthetic jets. AIAA Paper 2010-4582.

Zhong, S., Jabbal, M., TANG, H., Garcillan, L., Guo, F., Wood, N. \& Warsop, C. 2007 Towards the design of synthetic-jet actuators for full-scale flight conditions. Flow Turbul. Combust. 78 (3), 283-307.

ZHOU, J. \& ZHONG, S. 2009 Numerical simulation of the interaction of a circular synthetic jet with a boundary layer. Comput. Fluids 38 (2), 393-405. 